3 SAMPLE PREPARATION AND ANALYSES

4 Comendite, trachyte, latite, and trachybasalt whole pumices or scoria were

5 collected on the Chinese side of the border in 1989, 2005, and 2013 (Fig. 1, Table 1).

6 Results for the ME comendite focus on a single $\sim 0.5 \mathrm{~m}$ pumice (51) collected from a

7 tephra fall deposit and the comendite type section $\sim 26 \mathrm{~km}$ northeast of the caldera

8 and from two different tephra fall deposits located $\sim 33 \mathrm{~km}$ east of the caldera that

9 contain pebble-sized pumice and 3-20 mg intact potassium feldspar and

10 clinopyroxene crystals (BA and BB, Table 1). Trachyte results originate from

11 pumices collected on the northern flank of the caldera (ME TC-10-13 and TC-15-13)

12 and along the Chinese side of Tianchi shore (Baguamiao TC-24-13, Liuhaojie TC-27-

1313 L1, and TC-28-13 L2). ME trachybasalt scoria (CB1) was collected approximately

14 halfway up the northern flank of the volcano and ME latite pumice (95) was

15 collected $\sim 22 \mathrm{~km}$ east of the caldera.

16 Pumices of comendite and trachyte were hand crushed and sieved to minimize

17 crystal fragmentation. Potassium feldspar crystals and crystal-free pumice shards

18 were hand picked from sieve fractions $>0.355 \mathrm{~mm}$. Crystals were etched in $\sim 15 \%$

19 hydrofluoric acid to remove adhering glass and placed in a sonicating bath with

20 ultrafiltered water for $\sim 25$ minutes. Clean crystals were then hand picked to obtain

21 sanidine separates with minimal melt inclusions.

22 Three types of whole rock or pumice separates were prepared. Firstly, whole

23 pumice clasts with either high crystal contents (i.e., >10\%, TC 10-13, TC 15-13, TC

24 24-13, TC 27-13 and TC 28-13) or low crystal contents (i.e., <3\%, 51, 95, and CB1) 
25 were either powdered in a tungsten carbide shatterbox $(51,95$, and CB1) or in an

26 aluminum ball mill (TC 10-13, TC 15-13, TC 24-13, TC 27-13, and TC 28-13),

27 dissolved in hydrofluoric, nitric, and hydrochloric acids, and analyzed directly.

28 These are designated by WR (i.e., whole rocks) in Tables 1 and DR 1 . In addition,

29 crystal-free trachyte and comendite pumice separates were either directly dissolved

30 using hydrofluoric, nitric, and hydrochloric acids, and designated as CF Pumice (i.e.,

31 crystal-free pumice), or were sequentially dissolved using $10 \%$ hydrofluoric acid at

32 room temperature in 2 hour increments where any unpicked crystals remained

33 undissolved. The hydrofluoric acid solution containing the dissolved pumice was

34 decanted from any remaining crystals and additional nitric and hydrochloric acids

35 were added to ensure complete dissolution. These are designated as SD Pumice (i.e.,

36 sequential dissolution pumice). Major and trace element compositions of whole

37 pumice powders and crystal-free pumice powders are given in Table DR 1, and

38 average feldspar compositions of four sanidine crystals from each of the comendite

39 and selected trachytes are given in Table DR 2.

40 In contrast to pumice, selected clean sanidine crystals were loaded into

41 machined Al discs and irradiated for 30 (NM-266) or 20 (NM-274) minutes at the

42 USGS TRIGA Reactor in Denver (CO). Fish Canyon Tuff sanidine (FC-2) was used as a

43 flux monitor and assigned an age of $28.201 \mathrm{Ma}$ (Kuiper, 2008). $\mathrm{A}^{40} \mathrm{~K}$ decay constant

44 of $5.463 \mathrm{e}^{-10} /$ a was used (Min, 2000). Argon was measured using a ThermoScientific

45 ARGUS IV mass spectrometer (Jan) on line with an automated all metal extraction

46 system at New Mexico Tech. NM266 was subjected to an extended delay following

47 irradiation, thus a fixed value for ${ }^{37} \mathrm{Ar}$ based on a K/Ca ratio of 320 was used for data 
48 reduction. This value was verified by additional analyses (not presented here).

49 Variation of this assumed value by an order of magnitude impacts final age

50 assignments by $<1 \mathrm{ka}$. The multi-collector configuration used for analysis was: ${ }^{40} \mathrm{Ar}-$

$51 \mathrm{H} 1,{ }^{39} \mathrm{Ar}-\mathrm{Ax},{ }^{38} \mathrm{Ar}-\mathrm{L} 1,{ }^{37} \mathrm{Ar}-\mathrm{L} 2$, and ${ }^{36} \mathrm{Ar}-\mathrm{L} 3$. Amplifiers used for H1, L1, and L2

52 Faradays were $1 \mathrm{e}^{12} \mathrm{Ohm}$, the Ax Faraday was $1 \mathrm{e}^{13} \mathrm{Ohm}$, and $\mathrm{L} 3$ used a CDD ion

53 counter with a deadtime of $14 n s$. Samples were heated for 25-45 seconds using a

$5475 \mathrm{~W}$ Photon Machines $\mathrm{CO}_{2}$ laser. Reactive gases were removed during heating by

$55 \quad 30-45$ second reactions with a SAES NP-10 getter operated at 1.6A and a D-50 getter

56 operated at room temperature. Mass spectrometer sensitivity was $6 \mathrm{e}^{-17} \mathrm{~mol} / \mathrm{fA}$.

57 Typical total system blank and backgrounds were 1-3 $\pm 2-5 \%, 0.05-0.1 \pm 15 \%, 0.02$

$58 \pm 100 \%, 0.01 \pm 100 \%$, and $0.03 \pm 5 \%, \times 10^{-17}$ moles for masses $40,39,38,37$, and 36 ,

59 respectively. Correction factors for interfering reactions originate from long-term

60 monitoring of more lengthy irradiations. For the short irradiation times utilized

61 here, the impact of these corrections on age determinations are minimal. J-factors

62 were determined to precisions of $\sim \pm 0.1 \%$ and used $\mathrm{CO}_{2}$ laser fusions of at least 6-

63 crystals from multiple radial positions around irradiation trays.

64 Approximately $200 \mathrm{mg}$ of comendite, trachyte, latite, and trachybasalt whole

65 rock powder or crystal-free pumice, directly dissolved or sequentially dissolved,

66 were analyzed for uranium, thorium, and radium concentrations and isotope ratios

67 using isotope dilution and ${ }^{236} \mathrm{U},{ }^{229} \mathrm{Th}$, and ${ }^{228} \mathrm{Ra}$ spikes. $\mathrm{Sr}, \mathrm{Nd}$, and $\mathrm{Pb}$ isotope ratios

68 were also measured on these same samples (Table DR 1). U-series isotope dilution

69 and isotopic concentration measurements were undertaken using a

70 ThermoScientific NeptunePlus MC-ICPMS at NMSU. Additional Nd and Pb isotope 
71 ratios were determined using MC-ICPMS with $\mathrm{Nd}$ isotopes normalized to

$72{ }^{146} \mathrm{Nd} /{ }^{144} \mathrm{Nd}=0.7219$ and $\mathrm{Pb}$ isotopes normalized to NBS997 $\mathrm{Tl}=0.41892$, while $\mathrm{Sr}$

73 isotopes were measured using TIMS, normalized to ${ }^{86} \mathrm{Sr} /{ }^{88} \mathrm{Sr}=0.1194$.

74

75

76 Figure DR $1 .{ }^{40} \mathrm{Ar} /{ }^{39} \mathrm{Ar}$ age spectra of two multi-crystal aliquots ( $\mathrm{a}$ and $\mathrm{b}$ ) and fifteen

77 single sanidine crystals (c-q) from a single ME comendite pumice (51). ${ }^{40} \mathrm{Ar} /{ }^{39} \mathrm{Ar}$

78 ages range from $\sim 16$ ka to within error of eruption and many have flat spectra not 
79 normally observed for sanidines affected by excess argon.
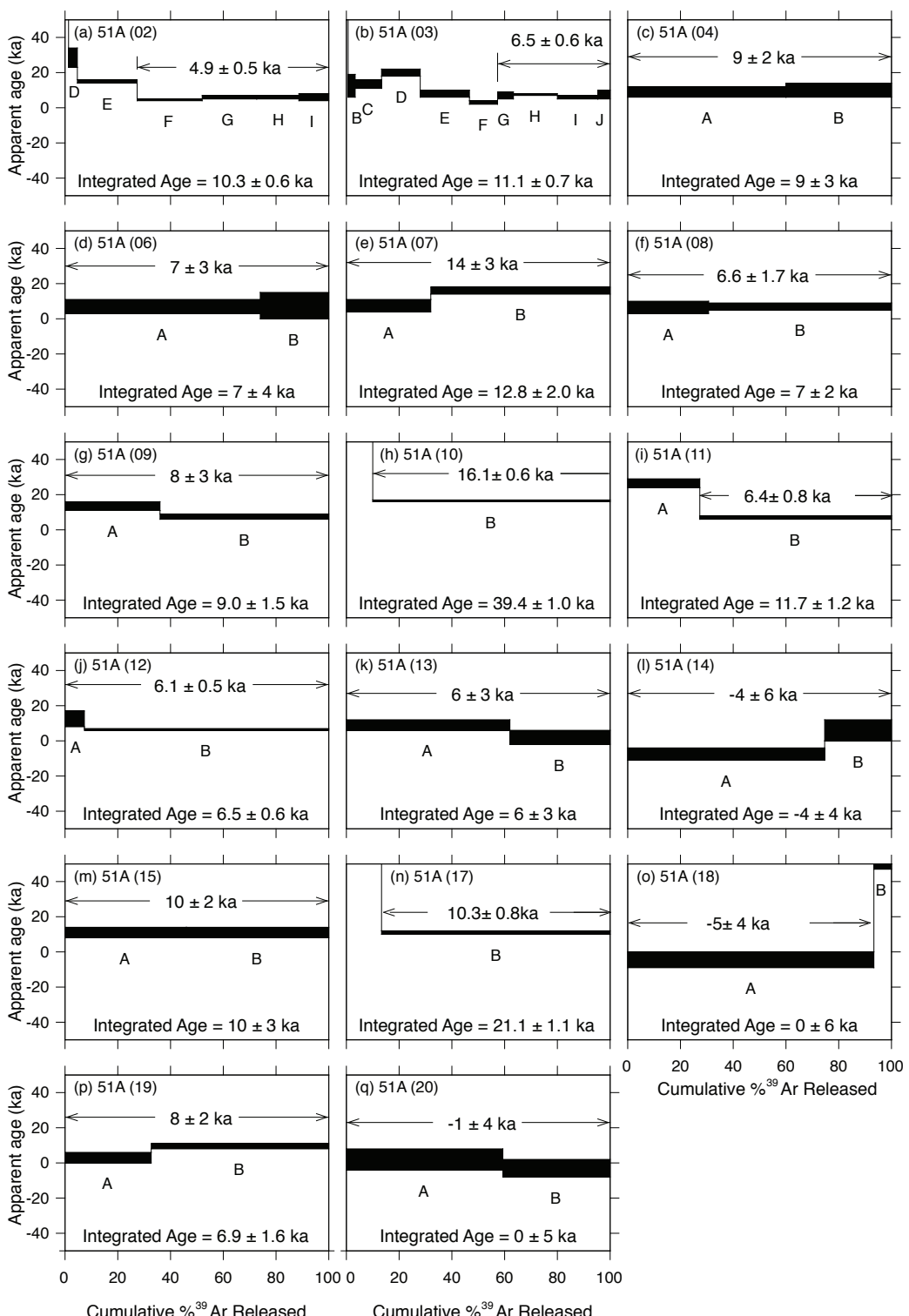

Cumulative $\%{ }^{39}$ Ar Released 
83 Figure DR 2. ${ }^{40} \mathrm{Ar} /{ }^{39} \mathrm{Ar}$ age spectra of two multi-crystal aliquots ( $\mathrm{a}$ and $\mathrm{b}$ ) and nine

84 single crystals analyses (c-k) from ME BA sanidines. Integrated ages are as old as

$85210 \mathrm{ka}$ whereas plateau segments are as young as $\sim 4 \mathrm{ka}$. All plateau ages are older

86 than the 946 CE eruption age.
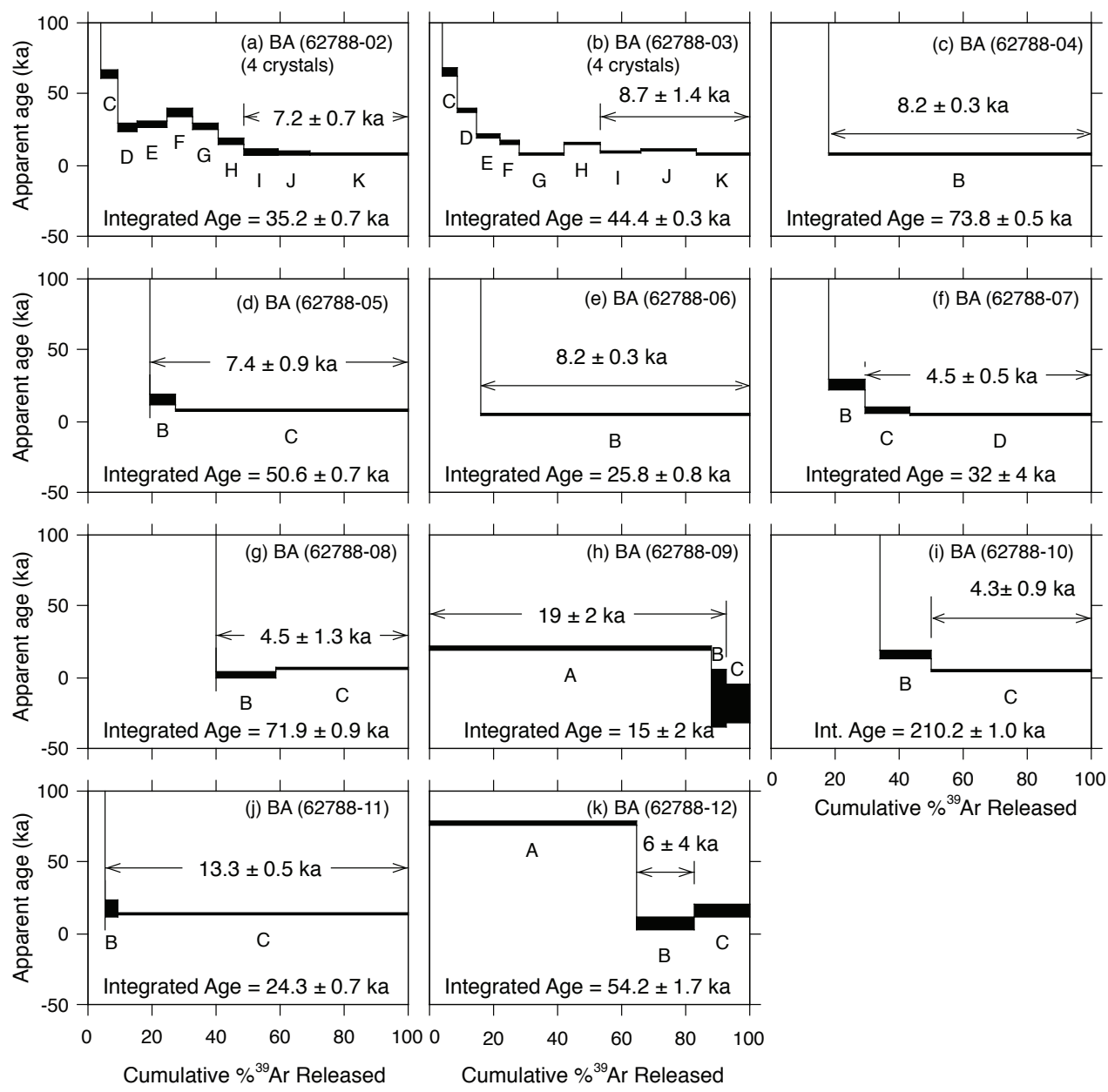
89 Figure DR 3. Example of ${ }^{40} \mathrm{Ar} /{ }^{39} \mathrm{Ar}$ age spectra of two multi-crystal aliquots (a and

90 b) and coarse and fine portions of single crystals subjected to hydrofluoric acid

91 etching (c-f) for sanidines obtained from distal portions (BB) of the ME comendite.

92 Sanidines are as young as $3.3 \pm 0.4 \mathrm{ka}$, however even with aggressive acid treatment

93 apparent ages remain older than the 946 CE eruption age.
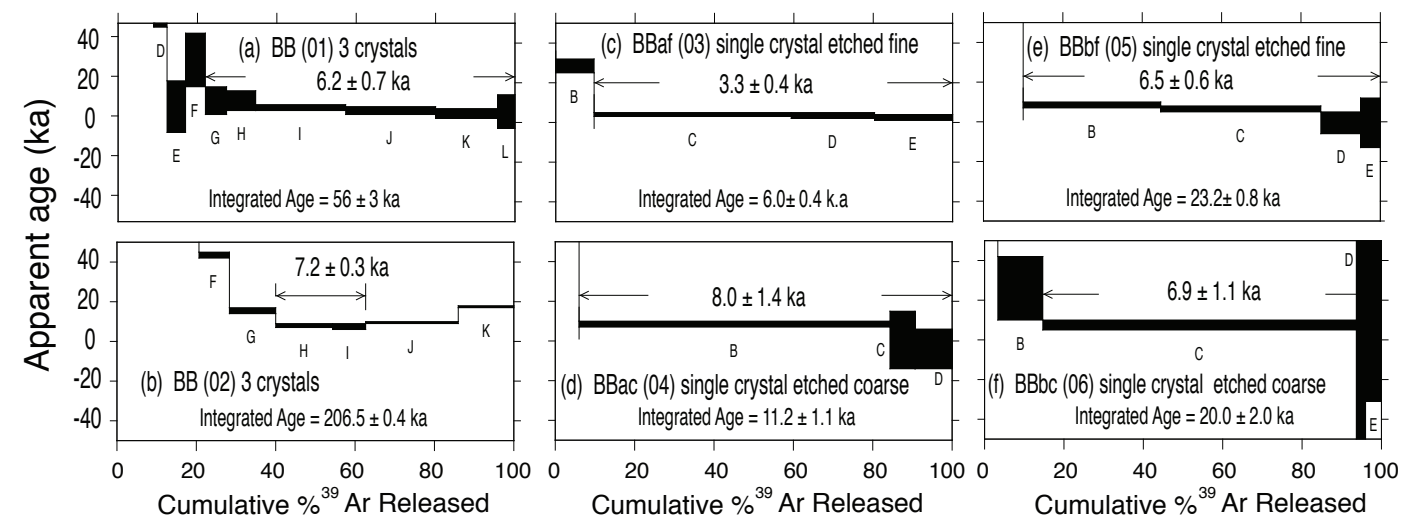

97 Figure DR 4. Single and multi-crystal ${ }^{40} \mathrm{Ar} /{ }^{39} \mathrm{Ar}$ age spectra of sanidines from the ME

98 trachtye. Plateau ages range from $4.1 \pm 0.8$ to $9.9 \pm 1.0 \mathrm{ka}$.
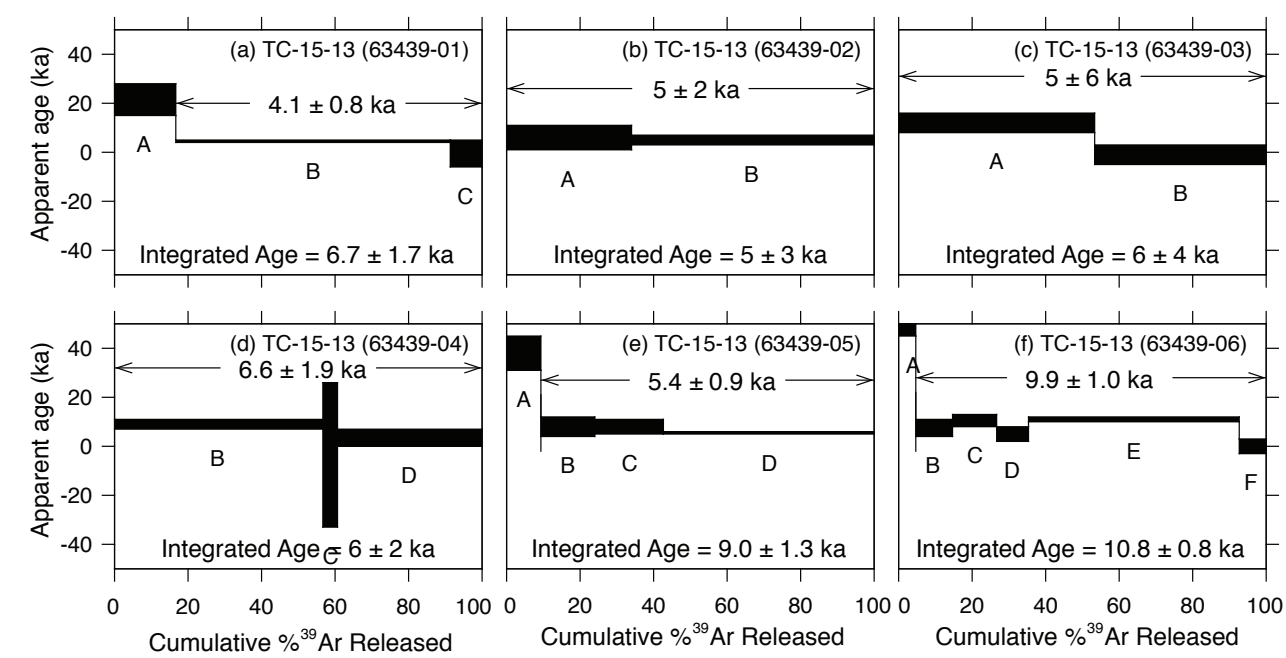

100

101 
102 Figure DR 5. Single and multi-crystal ${ }^{40} \mathrm{Ar} /{ }^{39} \mathrm{Ar}$ age spectra of sanidines from the

103 presumed 1668 CE Baguamiao trachyte. Plateau ages range from $2.8 \pm 0.6$ to $8.4 \pm$

$1040.4 \mathrm{ka}$.
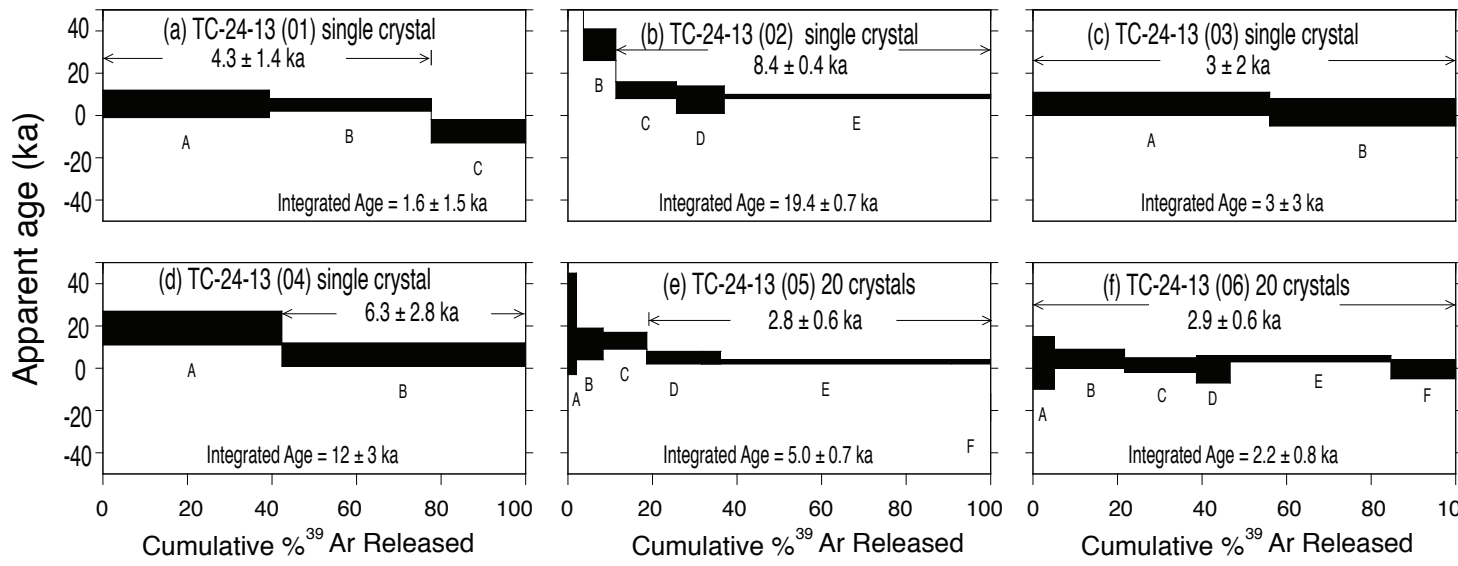

105
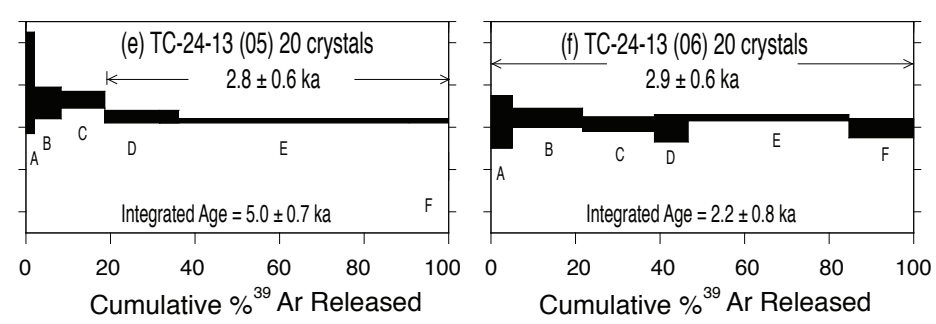

106

107

108

109 Figure DR 6. Single and multi-crystal ${ }^{40} \mathrm{Ar} /{ }^{39} \mathrm{Ar}$ age spectra of sanidines from the

110 presumed 1903 CE Liuhaojie trachyte. Plateau ages range from $4.2 \pm 0.9$ to $7.7 \pm 0.6$

$111 \mathrm{ka}$.

112
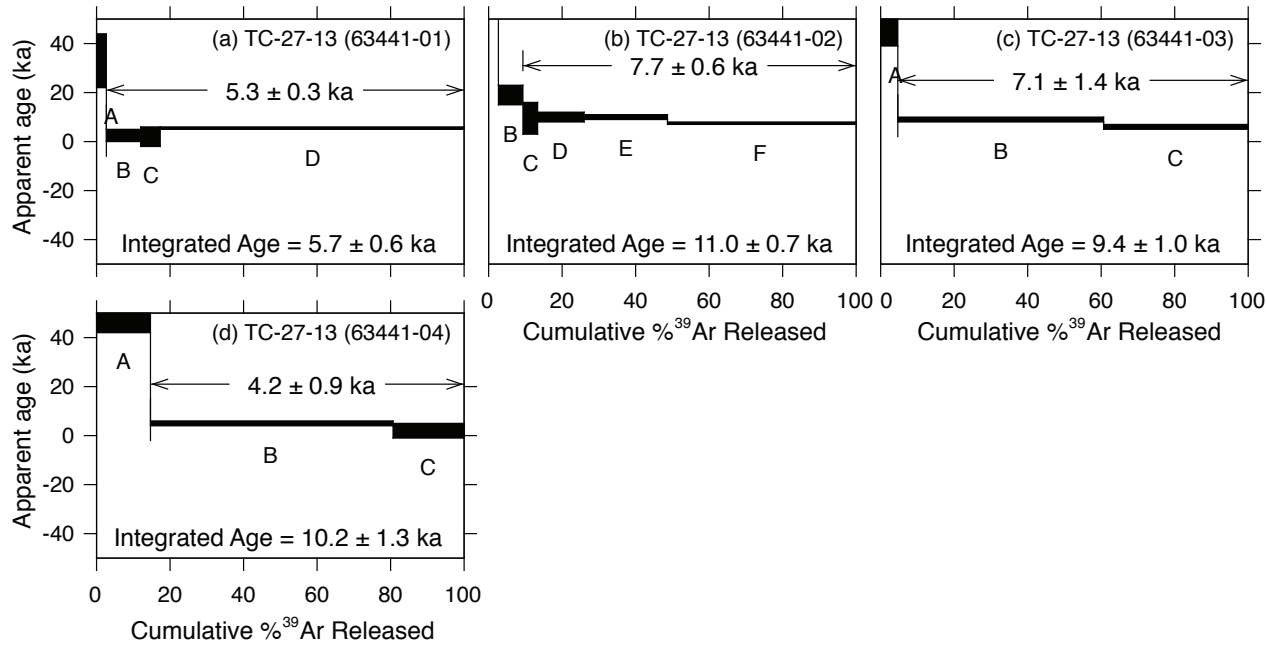


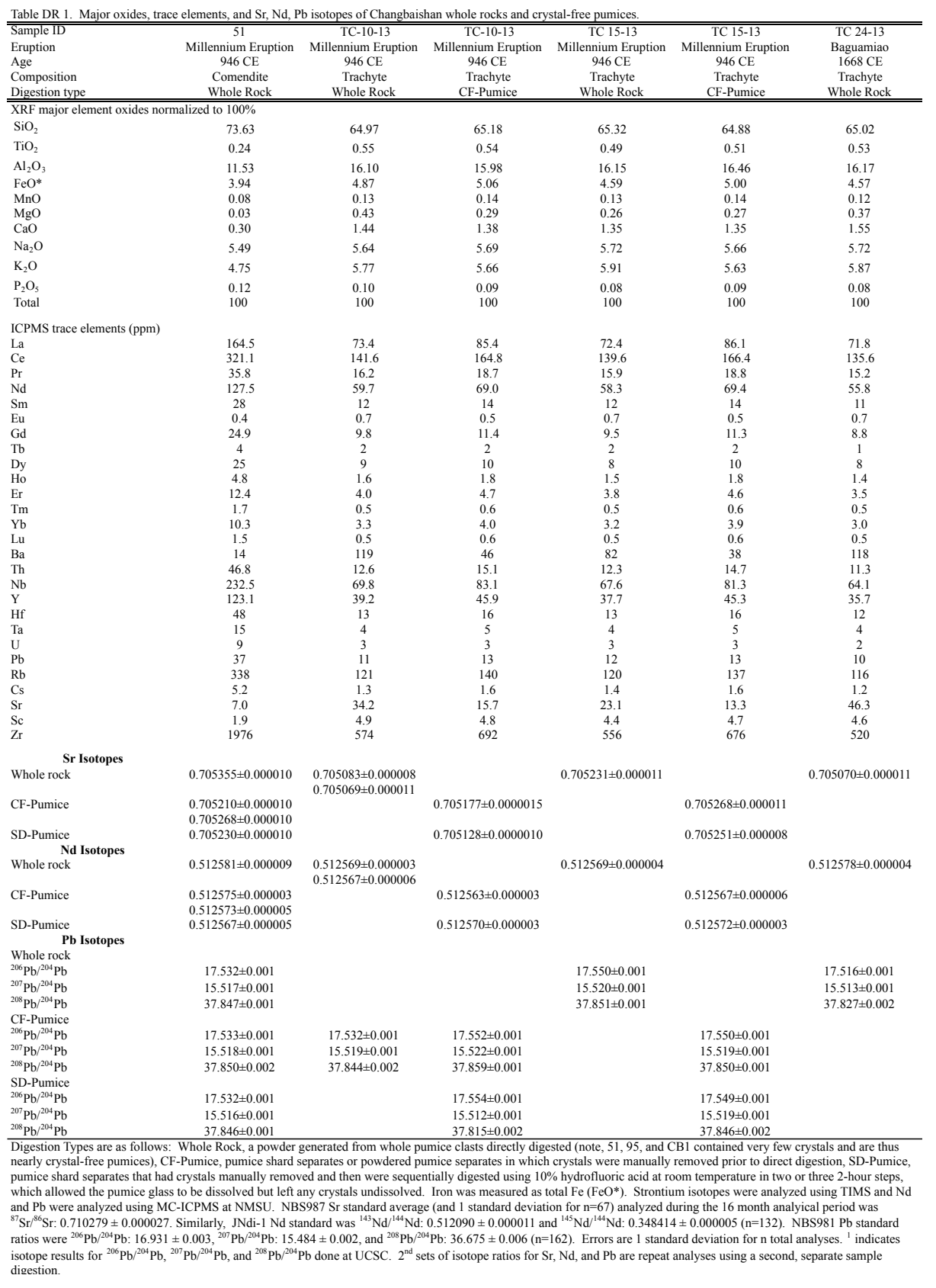

113 


\begin{tabular}{|c|c|c|c|c|c|c|}
\hline $\begin{array}{c}\text { TC 24-13 } \\
\text { Baguamiao } \\
1668 \mathrm{CE} \\
\text { Trachyte } \\
\text { CF-Pumice } \\
\end{array}$ & $\begin{array}{c}\text { TC 27-13 L1 } \\
\text { Liuhaojie } \\
1903 \text { CE } \\
\text { Trachyte L1 } \\
\text { Whole Rock } \\
\end{array}$ & $\begin{array}{l}\text { TC 27-13 L1 } \\
\text { Liuhaojie } \\
1903 \text { CE } \\
\text { Trachyte L1 } \\
\text { CF-Pumice } \\
\end{array}$ & $\begin{array}{c}\text { TC 28-13 L2 } \\
\text { Liuhaojie } \\
1903 \text { CE } \\
\text { Trachyte L2 } \\
\text { Whole Rock } \\
\end{array}$ & $\begin{array}{c}\text { TC 28-13 L2 } \\
\text { Liuhaojie } \\
1903 \text { CE } \\
\text { Trachyte L2 } \\
\text { CF-Pumice } \\
\end{array}$ & $\begin{array}{c}95 \\
\text { Millennium Eruption } \\
946 \mathrm{CE} \\
\text { Latite } \\
\text { Whole Rock } \\
\end{array}$ & $\begin{array}{c}\mathrm{CB} 1 \\
\text { Millennium Eruption } \\
946 \mathrm{CE} \\
\text { Trachybasalt } \\
\text { Whole Rock } \\
\end{array}$ \\
\hline 65.33 & 64.80 & 65.07 & 64.86 & 65.09 & 60.28 & 50.92 \\
\hline 0.49 & 0.50 & 0.54 & 0.47 & 0.54 & 1.24 & 3.08 \\
\hline $\begin{array}{l}16.09 \\
4.97\end{array}$ & $\begin{array}{l}16.65 \\
4.37\end{array}$ & $\begin{array}{l}15.83 \\
5.22\end{array}$ & $\begin{array}{c}16.70 \\
4.21\end{array}$ & $\begin{array}{l}15.94 \\
5.08\end{array}$ & $\begin{array}{l}16.66 \\
6.11\end{array}$ & $\begin{array}{l}17.12 \\
9.32\end{array}$ \\
\hline 0.14 & 0.12 & 0.14 & $\begin{array}{r}4.21 \\
0.11\end{array}$ & $\begin{array}{l}0.14 \\
0.14\end{array}$ & 0.13 & 0.13 \\
\hline 0.25 & 0.30 & 0.28 & 0.29 & 0.29 & 1.88 & 4.58 \\
\hline 1.29 & 1.46 & 1.45 & 1.50 & 1.45 & 3.10 & 8.13 \\
\hline 5.72 & 5.72 & 5.66 & 5.61 & 5.58 & 5.22 & 3.87 \\
\hline 5.65 & 5.99 & 5.72 & 6.17 & 5.79 & 4.58 & 2.22 \\
\hline 0.08 & 0.08 & 0.09 & 0.08 & 0.09 & 0.29 & 0.62 \\
\hline 100 & 100 & 100 & 100 & 100 & 100 & 100 \\
\hline 87.9 & 64.2 & 82.3 & 58.8 & 84.9 & 58.9 & 37.6 \\
\hline 173.7 & 122.4 & 155.6 & 110.8 & 162.3 & 113.1 & 76.1 \\
\hline 18.9 & 13.9 & 17.7 & 12.7 & 18.4 & 13.1 & 9.3 \\
\hline 68.5 & 51.3 & 64.9 & 46.9 & 68.0 & 49.6 & 38.2 \\
\hline 13 & 10 & 13 & 9 & 13 & 10 & 8 \\
\hline 0.5 & 0.8 & 0.5 & 0.9 & 0.5 & 2.4 & 2.9 \\
\hline 10.6 & 8.3 & 10.2 & 7.4 & 10.6 & 8.7 & 7.4 \\
\hline 2 & 1 & 2 & 1 & 2 & 1 & 1 \\
\hline 9 & 7 & 9 & 6 & 10 & 7 & 6 \\
\hline 1.7 & 1.3 & 1.7 & 1.2 & 1.8 & 1.3 & 1.0 \\
\hline 4.3 & 3.3 & 4.2 & 3.0 & 4.4 & 3.1 & 2.3 \\
\hline 0.6 & 0.5 & 0.6 & 0.4 & 0.6 & 0.4 & 0.3 \\
\hline 3.7 & 2.7 & 3.6 & 2.6 & 3.7 & 2.5 & 1.6 \\
\hline 0.6 & 0.4 & 0.6 & 0.4 & 0.6 & 0.4 & 0.2 \\
\hline 45 & 129 & 48 & 170 & 49 & 798 & 676 \\
\hline $\begin{array}{l}43.7 \\
\end{array}$ & 10.5 & $\begin{array}{l}40.7 \\
13.7\end{array}$ & 9.3 & 14.1 & 8.5 & 4.6 \\
\hline 82.2 & 59.5 & 77.6 & 52.3 & 79.3 & 50.1 & 38.3 \\
\hline 45.1 & 33.1 & 42.9 & 30.1 & 44.2 & 31.0 & 24.4 \\
\hline 15 & 11 & 14 & 10 & 14 & 9 & 6 \\
\hline 6 & 4 & 5 & 4 & 5 & 9 & 3 \\
\hline 3 & 2 & 3 & 2 & 3 & 2 & 1 \\
\hline 12 & 10 & 12 & 10 & 14 & 10 & 5 \\
\hline 140 & 110 & 130 & 102 & 137 & 85 & 39 \\
\hline 1.6 & 1.2 & 1.6 & 1.0 & 1.6 & 0.9 & 0.3 \\
\hline 13.7 & 43.6 & 18.7 & 50.6 & 20.1 & 352.0 & 823.0 \\
\hline \multirow{3}{*}{$\begin{array}{l}4.2 \\
678\end{array}$} & 4.3 & 4.5 & 4.1 & 4.7 & 9.7 & 19.9 \\
\hline & 480 & 636 & 427 & 652 & 379 & 260 \\
\hline & $0.705062 \pm 0.000010$ & & $0.705201 \pm 0.000014$ & & $0.704971 \pm 0.000010^{1}$ & $0.704920 \pm 0.000010^{1}$ \\
\hline \multirow{2}{*}{$\begin{array}{l}0.705171 \pm 0.000010 \\
0.705178 \pm 0.000010 \\
0.705258 \pm 0.000011\end{array}$} & & $0.705143 \pm 0.000015$ & & $0.705216 \pm 0.000013$ & & \\
\hline & $0.512570 \pm 0.000004$ & & $0.512576 \pm 0.000002$ & & $0.512586 \pm 0.000005$ & $0.512608 \pm 0.000005$ \\
\hline $0.512568 \pm 0.000004$ & & $0.512576 \pm 0.000004$ & & $0.512573 \pm 0.000003$ & & \\
\hline \multicolumn{7}{|l|}{$\begin{array}{l}0.512575 \pm 0.000004 \\
0.512575 \pm 0.000003\end{array}$} \\
\hline & $17.532 \pm 0.001$ & & $17.539 \pm 0.001$ & & $17.519 \pm 0.001$ & $17.514 \pm 0.001$ \\
\hline & $15.514 \pm 0.001$ & & $15.517 \pm 0.001$ & & $15.517 \pm 0.001$ & $15.519 \pm 0.001$ \\
\hline & $37.830 \pm 0.002$ & & $37.836 \pm 0.002$ & & $37.832 \pm 0.002$ & $37.859 \pm 0.002$ \\
\hline $17.536 \pm 0.001$ & & $17.524 \pm 0.001$ & & $17.540 \pm 0.001$ & & \\
\hline $15.514 \pm 0.001$ & & $15.515 \pm 0.001$ & & $15.513 \pm 0.001$ & & \\
\hline $37.826 \pm 0.002$ & & $37.830 \pm 0.002$ & & $37.824 \pm 0.002$ & & \\
\hline \multicolumn{7}{|l|}{$17.532 \pm 0.001$} \\
\hline $15.511 \pm 0.001$ & & & & & & \\
\hline $37.819 \pm 0.002$ & & & & & & \\
\hline
\end{tabular}

114 
Table DR 2. Major oxides (weight percent) of representative potassium feldspars determined by electron microprobe analysis.

\begin{tabular}{|c|c|c|c|c|c|c|c|c|c|c|c|c|c|c|c|}
\hline Sample ID & Composition & Crystal & $\mathrm{SiO}_{2}$ & $\mathrm{Al}_{2} \mathrm{O}_{3}$ & $\mathrm{CaO}$ & $\mathrm{FeO}$ & $\mathrm{SrO}$ & $\mathrm{BaO}$ & $\mathrm{Na}_{2} \mathrm{O}$ & $\mathrm{K}_{2} \mathrm{O}$ & Total & An & $\mathrm{Ab}$ & Or & $\mathrm{K} / \mathrm{Ca}$ \\
\hline \multirow[t]{4}{*}{ Liu. TC 27-13 L1 } & Trachyte & $1(\mathrm{kf} 2)$ & 64.64 & 19.97 & 0.93 & 0.17 & 0.02 & 0.01 & 6.21 & 7.11 & 99.05 & 0.045 & 0.54 & 0.41 & 8.9 \\
\hline & & $2(\mathrm{kf} 2)$ & 64.93 & 19.80 & 0.73 & 0.16 & 0.02 & 0.00 & 5.83 & 7.62 & 99.09 & 0.036 & 0.52 & 0.45 & 12.1 \\
\hline & & $3(\mathrm{kf} 2)$ & 65.22 & 19.37 & 0.34 & 0.22 & 0.02 & 0.01 & 6.29 & 7.42 & 98.87 & 0.017 & 0.55 & 0.43 & 25.3 \\
\hline & & $4(\mathrm{kf} 2)$ & 65.05 & 19.66 & 0.65 & 0.20 & 0.02 & 0.00 & 6.01 & 7.56 & 99.16 & 0.032 & 0.53 & 0.44 & 13.4 \\
\hline \multirow[t]{4}{*}{ Bag. TC $24-13$} & Trachyte & $1(\mathrm{kf} 2)$ & 64.43 & 20.02 & 0.90 & 0.15 & 0.03 & 0.05 & 5.98 & 7.53 & 99.09 & 0.043 & 0.52 & 0.43 & 9.7 \\
\hline & & $2(\mathrm{kf} 2)$ & 64.75 & 19.98 & 0.85 & 0.18 & 0.02 & 0.03 & 6.03 & 7.43 & 99.27 & 0.041 & 0.53 & 0.43 & 10.2 \\
\hline & & $3(\mathrm{kf} 2)$ & 65.33 & 19.41 & 0.35 & 0.22 & 0.01 & 0.01 & 6.34 & 7.49 & 99.17 & 0.017 & 0.55 & 0.43 & 24.9 \\
\hline & & $4(\mathrm{kf} 2)$ & 65.08 & 19.96 & 0.72 & 0.15 & 0.01 & 0.02 & 6.06 & 7.69 & 99.69 & 0.035 & 0.53 & 0.44 & 12.4 \\
\hline \multirow[t]{4}{*}{ ME TC $15-13$} & Trachyte & $1(\mathrm{kf} 2)$ & 65.10 & 20.23 & 0.91 & 0.15 & 0.03 & 0.02 & 6.30 & 7.32 & 100.07 & 0.043 & 0.54 & 0.41 & 9.3 \\
\hline & & $2(\mathrm{kf} 2)$ & 65.13 & 20.23 & 0.98 & 0.16 & 0.02 & 0.01 & 6.14 & 7.47 & 100.14 & 0.047 & 0.53 & 0.42 & 8.8 \\
\hline & & $3(\mathrm{kf} 2)$ & 66.29 & 19.49 & 0.24 & 0.23 & 0.01 & 0.01 & 6.86 & 6.96 & 100.09 & 0.012 & 0.59 & 0.40 & 33.5 \\
\hline & & $4(\mathrm{kf} 2)$ & 65.23 & 20.08 & 0.81 & 0.16 & 0.04 & 0.01 & 5.88 & 7.94 & 100.14 & 0.039 & 0.51 & 0.45 & 11.4 \\
\hline \multirow[t]{4}{*}{ ME 51} & Comendite & $1(\mathrm{kf1})$ & 67.14 & 18.92 & 0.01 & - & - & - & 7.14 & 6.50 & 99.71 & 0.0005 & 0.63 & 0.37 & 754.5 \\
\hline & & $2(\mathrm{kfl})$ & 67.15 & 19.00 & 0.01 & - & - & - & 7.23 & 6.55 & 99.95 & 0.0005 & 0.63 & 0.37 & 760.3 \\
\hline & & $3(\mathrm{kfl})$ & 67.36 & 18.87 & 0.01 & - & - & - & 7.28 & 6.45 & 99.97 & 0.0005 & 0.63 & 0.37 & 748.7 \\
\hline & & $4(\mathrm{kfl})$ & 67.02 & 18.80 & 0.01 & - & - & - & 7.13 & 6.28 & 99.24 & 0.0005 & 0.63 & 0.37 & 729.0 \\
\hline
\end{tabular}

5, Or $>40$, and Ab 50-60. Electron microprobe analyses done at Oregon State University and New Mexico Bureau of Geology and Mineral Resources.

Table DR 3. ${ }^{40} \mathrm{Ar} /{ }^{39} \mathrm{Ar}$ results for young Changbaishan sanidines from comendite and trachyte deposits.

\begin{tabular}{|c|c|c|c|c|c|c|c|c|c|c|c|}
\hline \multicolumn{3}{|c|}{ Analysis Identifiers } & \multicolumn{6}{|c|}{ Preferred Age } & \multicolumn{3}{|c|}{ Integrated Age } \\
\hline Sample & L\# & Irrad & Analysis & $\mathrm{n}$ & $\%{ }^{39} \mathrm{Ar}$ & MSWD & Age (ka) & $\pm 1 \sigma$ & $\mathrm{n}$ & Age (ka) & $\pm 1 \sigma$ \\
\hline \multicolumn{12}{|c|}{ ME Comendite } \\
\hline 51 & 62784-02 & NM-266A & $\mathrm{Pt}$ & 4 & 72.5 & 1.0 & 4.95 & \pm 0.49 & 8 & 10.3 & \pm 0.6 \\
\hline 51 & $62784-03$ & NM-266A & $\mathrm{Pt}$ & 4 & 42.1 & 0.3 & 6.53 & \pm 0.58 & 10 & 11.1 & \pm 0.7 \\
\hline 51 & 62784-04 & NM-266A & $\mathrm{Pt}$ & 2 & 100.0 & 0.0 & 8.8 & \pm 2.3 & 2 & 9.0 & \pm 3.0 \\
\hline 51 & 62784-06 & NM-266A & $\mathrm{Pt}$ & 2 & 100.0 & 0.0 & 6.7 & \pm 3.4 & 2 & 7.0 & \pm 4.0 \\
\hline 51 & 62784-07 & NM-266A & $\mathrm{Pt}$ & 2 & 100.0 & 4.8 & 14.1 & \pm 3.3 & 2 & 12.8 & \pm 2.0 \\
\hline 51 & $62784-08$ & NM-266A & $\mathrm{Pt}$ & 2 & 100.0 & 0.0 & 6.6 & \pm 1.7 & 2 & 7.0 & \pm 2.0 \\
\hline 51 & 62784-09 & NM-266A & $\mathrm{Pt}$ & 2 & 100.0 & 5.2 & 8.1 & \pm 2.5 & 2 & 9.0 & \pm 1.5 \\
\hline 51 & $62784-10$ & NM-266A & Step B & 1 & 89.8 & 0.0 & 16.10 & \pm 0.60 & 2 & 39.4 & \pm 1.0 \\
\hline 51 & $62784-11$ & NM-266A & Step B & 1 & 72.5 & 0.0 & 6.40 & \pm 0.80 & 2 & 11.7 & \pm 1.2 \\
\hline 51 & $62784-12$ & NM-266A & $\mathrm{Pt}$ & 2 & 100.0 & 1.9 & 6.12 & \pm 0.49 & 2 & 6.5 & \pm 0.6 \\
\hline 51 & $62784-13$ & NM-266A & $\mathrm{Pt}$ & 2 & 100.0 & 1.9 & 5.9 & \pm 3.2 & 2 & 6.0 & \pm 3.0 \\
\hline 51 & $62784-14$ & NM-266A & $\mathrm{Pt}$ & 2 & 100.0 & 3.6 & -4.2 & \pm 5.8 & 2 & -4.0 & \pm 4.0 \\
\hline 51 & $62784-15$ & NM-266A & $\mathrm{Pt}$ & 2 & 100.0 & 0.0 & 10.4 & \pm 2.1 & 2 & 10.0 & \pm 3.0 \\
\hline 51 & 62784-17 & NM-266A & Step B & 1 & 86.4 & 0.0 & 10.3 & \pm 0.8 & 2 & 21.1 & \pm 1.1 \\
\hline 51 & $62784-18$ & NM-266A & Step A & 1 & 93.5 & 0.0 & -5.0 & \pm 4.0 & 2 & 0.0 & \pm 6.0 \\
\hline 51 & $62784-19$ & NM-266A & $\mathrm{Pt}$ & 2 & 100.0 & 4.0 & 7.9 & \pm 2.3 & 2 & 6.9 & \pm 1.6 \\
\hline 51 & $62784-20$ & NM-266A & $\mathrm{Pt}$ & 2 & 100.0 & 0.4 & -1.4 & \pm 3.7 & 2 & 0.0 & \pm 5.0 \\
\hline \multicolumn{12}{|c|}{ ME Comendite } \\
\hline BB & 62789-01 & NM-266B & $\mathrm{Pt}$ & 6 & 77.8 & 2.0 & 6.20 & \pm 0.73 & 12 & 56.0 & \pm 3.0 \\
\hline BB & 62789-02 & NM-266B & $\mathrm{Pt}$ & 2 & 22.8 & 0.4 & 7.16 & \pm 0.33 & 10 & 207.0 & \pm 0.4 \\
\hline BBaf & $62789-03$ & NM-266B & $\mathrm{Pt}$ & 3 & 90.3 & 1.9 & 3.32 & \pm 0.39 & 5 & 6.0 & \pm 0.4 \\
\hline $\mathrm{BBac}$ & 62789-04 & NM-266B & $\mathrm{Pt}$ & 3 & 93.9 & 4.0 & 7.98 & \pm 1.36 & 4 & 11.2 & \pm 1.1 \\
\hline $\mathrm{BBbf}$ & 62789-05 & NM-266B & $\mathrm{Pt}$ & 4 & 89.9 & 4.2 & 6.49 & \pm 0.60 & 5 & 23.2 & \pm 0.8 \\
\hline $\mathrm{BBbc}$ & $62789-06$ & NM-266B & $\mathrm{Pt}$ & 4 & 96.4 & 1.8 & 6.90 & \pm 1.10 & 5 & 20.0 & \pm 2.0 \\
\hline \multicolumn{12}{|c|}{ ME Comendite } \\
\hline BA & $62788-02$ & NM-266B & $\mathrm{Pt}$ & 3 & 49.3 & 0.5 & 7.16 & \pm 0.65 & 11 & 35.2 & \pm 0.7 \\
\hline BA & $62788-03$ & NM-266B & $\mathrm{Pt}$ & 3 & 46.7 & 24.3 & 8.73 & \pm 1.38 & 10 & 44.4 & \pm 0.3 \\
\hline $\mathrm{BA}$ & $62788-04$ & NM-266B & Step B & 1 & 81.7 & na & 8.19 & \pm 0.28 & 2 & 73.8 & \pm 0.5 \\
\hline BA & $62788-05$ & NM-266B & $\mathrm{Pt}$ & 2 & 80.5 & 4.5 & 7.41 & \pm 0.88 & 3 & 50.6 & \pm 0.7 \\
\hline BA & $62788-06$ & NM-266B & Step B & 1 & 83.5 & na & 3.59 & \pm 0.40 & 2 & 25.8 & \pm 0.8 \\
\hline BA & $62788-07$ & NM-266B & $\mathrm{Pt}$ & 2 & 70.3 & 0.9 & 4.54 & \pm 0.52 & 4 & 32.0 & \pm 4.0 \\
\hline BA & $62788-08$ & NM-266B & $\mathrm{Pt}$ & 2 & 59.5 & 2.1 & 4.5 & \pm 1.3 & 3 & 71.9 & \pm 0.9 \\
\hline BA & 62788-09 & NM-266B & $\mathrm{Pt}$ & 2 & 93.0 & 3.0 & 19.2 & \pm 2.5 & 3 & 15.0 & \pm 2.0 \\
\hline BA & $62788-10$ & NM-266B & Step C & 1 & 49.8 & na & 4.3 & \pm 0.9 & 3 & 210.2 & \pm 1.0 \\
\hline BA & $62788-11$ & NM-266B & $\mathrm{Pt}$ & 2 & 94.3 & 0.4 & 13.28 & \pm 0.54 & 3 & 24.3 & \pm 0.7 \\
\hline BA & $62788-12$ & NM-266B & Step B & 1 & 17.9 & na & 6.2 & \pm 4.3 & 3 & 54.2 & \pm 1.7 \\
\hline \multicolumn{12}{|c|}{ ME Trachyte } \\
\hline TC-15-13 & 63439-01 & NM-274B & $\mathrm{Pt}$ & 2 & 83.1 & 0.7 & 4.05 & \pm 0.83 & 3 & 6.70 & \pm 1.35 \\
\hline TC-15-13 & 63439-02 & NM-274B & $\mathrm{Pt}$ & 2 & 100.0 & 0.1 & 4.7 & \pm 2.1 & 2 & 4.87 & \pm 2.30 \\
\hline TC-15-13 & 63439-03 & NM-274B & $\mathrm{Pt}$ & 2 & 100.0 & 4.7 & 5.0 & \pm 6.4 & 2 & 5.59 & \pm 2.96 \\
\hline TC-15-13 & 63439-04 & NM-274B & $\mathrm{Pt}$ & 3 & 100.0 & 1.1 & 6.6 & \pm 1.9 & 3 & 5.79 & \pm 2.13 \\
\hline TC-15-13 & 63439-05 & NM-274B & $\mathrm{Pt}$ & 3 & 90.5 & 0.5 & 5.41 & \pm 0.85 & 4 & 8.99 & \pm 1.13 \\
\hline TC-15-13 & 63439-06 & NM-274B & $\mathrm{Pt}$ & 5 & 95.1 & 3.8 & 9.9 & \pm 1.0 & 6 & 10.78 & \pm 0.74 \\
\hline \multicolumn{12}{|c|}{ Baguamiao Trachyte } \\
\hline TC-24-13 & $63440-01$ & NM-274B & $\mathrm{Pt}$ & 2 & 77.8 & 0.0 & 4.3 & \pm 3.5 & 3 & 1.6 & \pm 1.5 \\
\hline TC-24-13 & $63440-02$ & NM-274B & $\mathrm{Pt}$ & 3 & 88.7 & 1.2 & 8.38 & \pm 0.46 & 5 & 19.36 & \pm 0.68 \\
\hline TC-24-13 & $63440-03$ & NM-274B & $\mathrm{Pt}$ & 2 & 100.0 & 1.0 & 3.5 & \pm 2.1 & 2 & 3.5 & \pm 2.1 \\
\hline TC-24-13 & $63440-04$ & NM-274B & Step B & 1 & 57.6 & NA & 6.3 & \pm 2.8 & 2 & 11.5 & \pm 2.4 \\
\hline TC-24-13 & $63440-05$ & NM-274B & $\mathrm{Pt}$ & 2 & 79.2 & 1.3 & 2.82 & \pm 0.58 & 5 & 5.00 & \pm 0.60 \\
\hline TC-24-13 & $63440-06$ & NM-274B & $\mathrm{Pt}$ & 6 & 100.0 & 1.5 & 2.95 & \pm 0.77 & 6 & 2.24 & \pm 0.76 \\
\hline \multicolumn{12}{|c|}{ Liuhaojie Trachyte } \\
\hline TC-27-13 & 63441-01 & NM-274B & $\mathrm{Pt}$ & 3 & 96.8 & 1.2 & 5.31 & \pm 0.32 & 4 & 5.72 & \pm 0.54 \\
\hline TC-27-13 & 63441-02 & NM-274B & $\mathrm{Pt}$ & 4 & 90.1 & 1.7 & 7.72 & \pm 0.60 & 6 & 10.97 & \pm 0.65 \\
\hline TC-27-13 & 63441-03 & NM-274B & $\mathrm{Pt}$ & 2 & 94.7 & 5.8 & 7.1 & \pm 1.4 & 3 & 9.38 & \pm 0.80 \\
\hline TC-27-13 & 63441-04 & NM-274B & $\mathrm{Pt}$ & 2 & 85.2 & 0.8 & 4.23 & \pm 0.90 & 3 & 10.2 & \pm 1.3 \\
\hline
\end{tabular}

for preferred age, $\mathrm{n}$ : number of steps used to calculate the preferred age, $\%{ }^{39} \mathrm{Ar}$ : percentage of ${ }^{39} \mathrm{Ar}$ comprising the preferred age, MSWD: Mean Square Weighted Deviation, 1ఠ: 1 sigma errors calculated for ages, n: number of steps used to calculate the the integrated age, af or bf: aliquo 


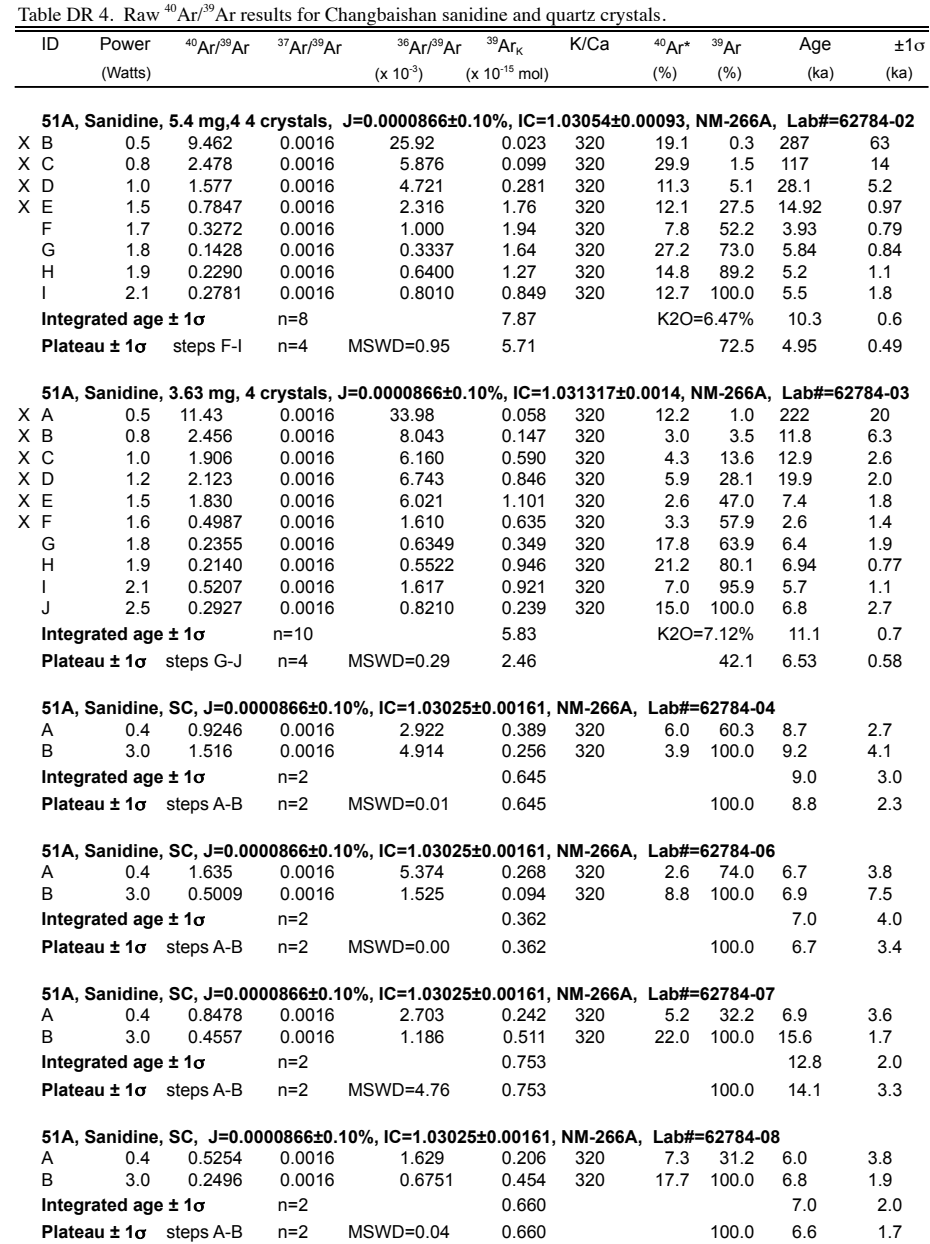

118 


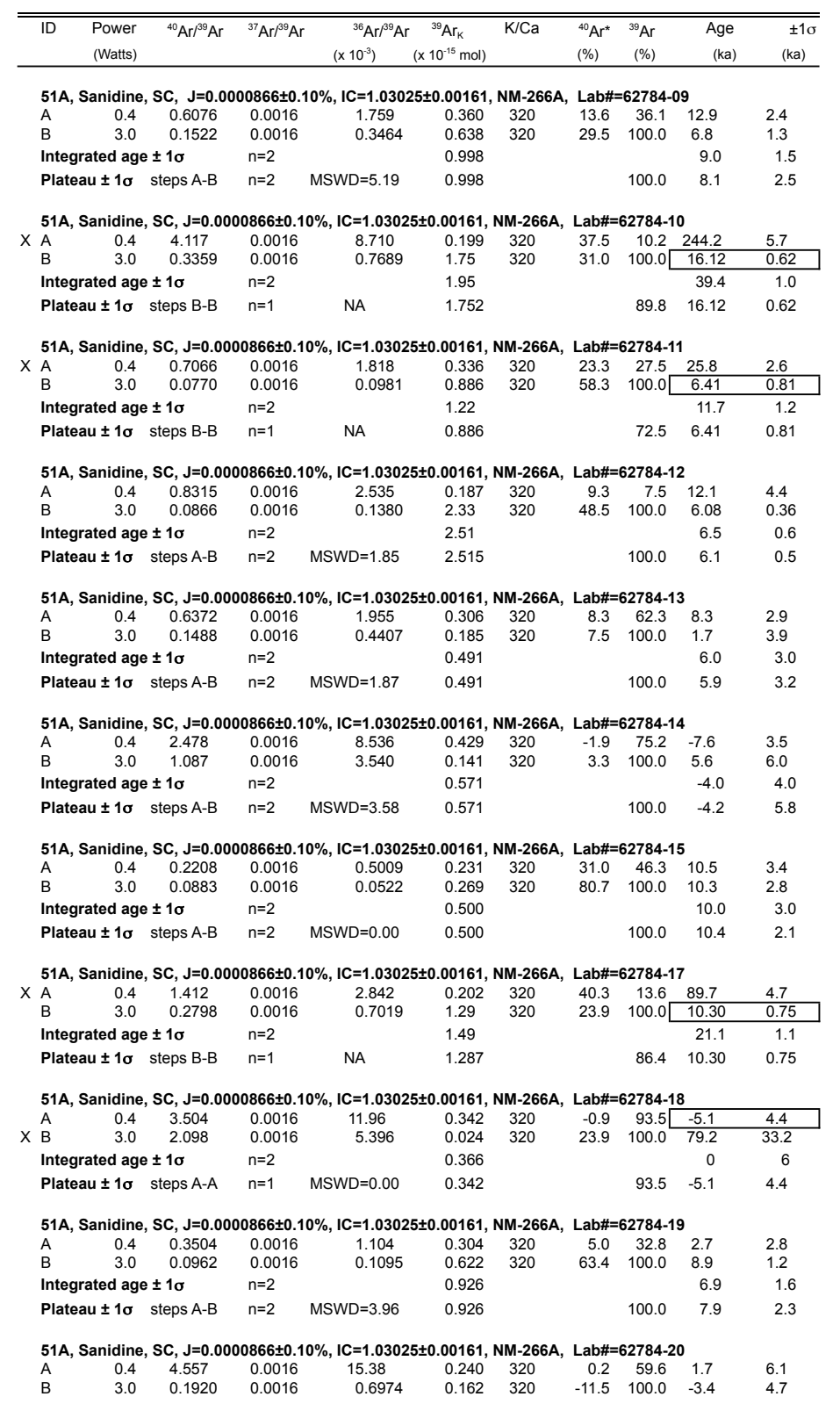




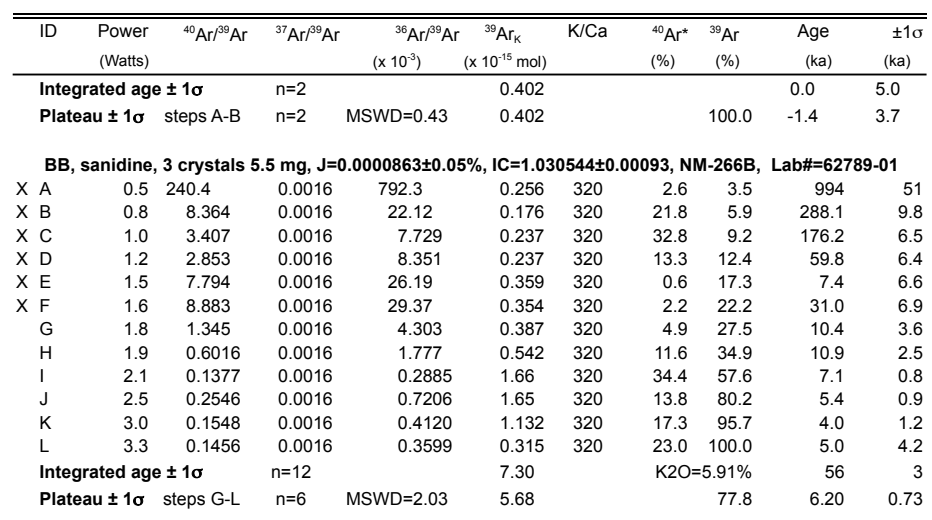

\begin{tabular}{|c|c|c|c|c|c|c|c|c|c|c|}
\hline \multicolumn{11}{|c|}{ 0014, NM-266B， Lab\#=62789-02 } \\
\hline$x \mathrm{~A}$ & 0.5 & 41.03 & 0.0016 & 92.09 & 0.142 & 320 & 33.7 & 1.2 & 2180 & 20 \\
\hline B & 0.8 & 14.08 & 0.0016 & 7.158 & 0.300 & 320 & 85.0 & 3.8 & 1887.6 & 5.4 \\
\hline & 1.0 & 12.29 & 0.0016 & 2.482 & 0.632 & 320 & 94.0 & 9.2 & 1822.8 & 2.5 \\
\hline & 1.5 & 1.699 & 0.0016 & 1.800 & 1.37 & 320 & 68.5 & 20.9 & 183.12 & 0.94 \\
\hline & 1.6 & 0.4124 & 0.0016 & 0.4523 & 0.878 & 320 & 66.9 & 28.5 & 42.81 & 0.80 \\
\hline & 1.8 & 0.1648 & 0.0016 & 0.2108 & 1.35 & 320 & 60.4 & 40.1 & 15.00 & 0.52 \\
\hline $\mathrm{H}$ & 1.9 & 0.0805 & 0.0016 & 0.0906 & 1.67 & 320 & 63.2 & 54.3 & 7.27 & 0.38 \\
\hline I & 2.1 & 0.0670 & 0.0016 & 0.0555 & 0.991 & 320 & 72.7 & 62.8 & 6.82 & 0.66 \\
\hline & 2.5 & 0.0993 & 0.0016 & 0.1079 & 2.71 & 320 & 65.3 & 86.1 & 9.45 & 0.26 \\
\hline & 3.0 & 0.1472 & 0.0016 & 0.1098 & 1.62 & 320 & 76.7 & 100.0 & 16.91 & 0.40 \\
\hline \multicolumn{3}{|c|}{ Integrated ag } & $n=10$ & & 11.67 & & $\mathrm{~K} 2 \mathrm{O}$ & $6.92 \%$ & 206.5 & 0.4 \\
\hline
\end{tabular}

\begin{tabular}{|c|c|c|c|c|c|c|c|c|c|c|}
\hline \\
\hline$x \mathrm{~A}$ & 0.3 & 6.706 & 0.0016 & 19.64 & 0.020 & 320 & 13.5 & 0.3 & 142.9 & 53.9 \\
\hline$x B$ & 0.7 & 0.5468 & 0.0016 & 1.222 & 0.702 & 320 & 33.1 & 9.7 & 28.2 & 1.7 \\
\hline C & 1.5 & 0.1664 & 0.0016 & 0.4589 & 3.69 & 320 & 14.6 & 59.5 & 3.67 & 0.35 \\
\hline D & 2.0 & 0.0643 & 0.0016 & 0.1256 & 1.56 & 320 & 34.6 & 80.5 & 3.10 & 0.69 \\
\hline $\mathrm{E}$ & 3.0 & 0.0584 & 0.0016 & 0.1256 & 1.45 & 320 & 27.2 & 100.0 & 2.18 & 0.69 \\
\hline \multicolumn{3}{|c|}{ Integrated age $\pm 1 \sigma$} & $n=5$ & & 7.41 & & $\mathrm{~K} 2 \mathrm{O}=$ & $=6.09 \%$ & 6.0 & 0.4 \\
\hline \multicolumn{3}{|l|}{ Plate } & $n=3$ & $W D=1.93$ & 6.69 & & & 90.3 & 3.32 & 0.39 \\
\hline
\end{tabular}

\begin{tabular}{|c|c|c|c|c|c|c|c|c|c|c|}
\hline \multicolumn{11}{|c|}{ BBac, sanidine, SC, etched, coarse, $1.42 \mathrm{mg}, \mathrm{J}=0.0000863 \pm 0.05 \%$, IC $=1.03128 \pm 0.00104, \mathrm{NM}-266 \mathrm{~B}$, Lab\#=62789-04 } \\
\hline A & 0.6 & 1.198 & 0.0016 & 2.200 & 0.123 & 320 & 45.6 & 6.1 & 85.7 & 8.3 \\
\hline B & 1.5 & 0.1755 & 0.0016 & 0.3903 & 1.59 & 320 & 31.3 & 84.4 & 8.30 & 0.69 \\
\hline C & 2.0 & 0.0080 & 0.0016 & 0.0066 & 0.131 & 320 & -399.7 & 90.8 & -0.3 & 7.1 \\
\hline D & 3.0 & 0.0377 & 0.0016 & 0.2018 & 0.187 & 320 & -101.5 & 100.0 & -4.8 & 5.1 \\
\hline \multicolumn{3}{|c|}{ Integrated age $\pm 1 \sigma$} & \multicolumn{2}{|l|}{$n=4$} & 2.04 & & \multicolumn{2}{|c|}{$\mathrm{K} 2 \mathrm{O}=6.38 \%$} & 11.2 & 1.1 \\
\hline & $\pm 1 \sigma$ & eps B-D & $n=3$ & $M S W D=3.98$ & 1.913 & & & 93.9 & 8.0 & 1.4 \\
\hline
\end{tabular}

BBbf, sanidine, SC, etched, fine, $2.22 \mathrm{mg}, \mathrm{J}=0.0000863 \pm 0.05 \%$, IC $=1.03128 \pm 0.00104$, NM-266B, Lab\#=62789-05

\begin{tabular}{|c|c|c|c|c|c|c|c|c|c|c|}
\hline$x \mathrm{~A}$ & 0.5 & 2.386 & 0.0016 & 4.173 & 0.320 & 320 & 48.2 & 10.1 & 181.1 & 3.8 \\
\hline B & 0.8 & 0.1532 & 0.0016 & 0.3175 & 1.106 & 320 & 35.4 & 44.7 & 8.15 & 0.88 \\
\hline c & 1.5 & 0.2338 & 0.0016 & 0.6429 & 1.28 & 320 & 16.0 & 85.1 & 5.72 & 0.85 \\
\hline $\mathrm{D}$ & 2.0 & 0.0367 & 0.0016 & 0.1186 & 0.319 & 320 & -20.7 & 95.1 & -1.0 & 2.9 \\
\hline $\mathrm{E}$ & 3.0 & 0.0856 & 0.0016 & 0.2816 & 0.157 & 320 & -8.3 & 100.0 & -1.0 & 6.1 \\
\hline \multicolumn{3}{|c|}{ Integrated age $\pm 1 \sigma$} & $n=5$ & & 3.19 & & $\mathrm{~K} 2 \mathrm{O}=$ & $6.39 \%$ & 23.20 & 0.80 \\
\hline
\end{tabular}

BBbc, sanidine, SC, etched, coarse, $0.71 \mathrm{mg}, \mathrm{J}=0.0000863 \pm 0.05 \%$, IC=1.03128 $\pm 0.00104, \mathrm{NM}-266 \mathrm{~B}$, Lab\#=62789-06 


\begin{tabular}{|c|c|c|c|c|c|c|c|c|c|c|}
\hline ID & Power & ${ }^{40} \mathrm{Ar} /{ }^{39} \mathrm{Ar}$ & ${ }^{37} \mathrm{Ar}{ }^{39} \mathrm{Ar}$ & ${ }^{36} \mathrm{Ar}{ }^{39} \mathrm{Ar}$ & ${ }^{3}{ }^{39} \mathrm{Ar}_{\mathrm{K}}$ & $\mathrm{K} / \mathrm{Ca}$ & ${ }^{40} \mathrm{Ar}^{*}$ & ${ }^{39} \mathrm{Ar}$ & Age & $\pm 1 \sigma$ \\
\hline & (Watts) & & & $\left(\times 10^{-3}\right)$ & $\left(\times 10^{-15} \mathrm{~mol}\right)$ & & $(\%)$ & $(\%)$ & (ka) & (ka) \\
\hline$\times \mathrm{A}$ & 0.5 & 3.304 & 0.0016 & 4.468 & 0.038 & 320 & 60.1 & 3.6 & 313 & 26 \\
\hline$x B$ & 0.8 & 0.2329 & 0.0016 & 0.2244 & 0.117 & 320 & 70.9 & 14.8 & 25.2 & 8.0 \\
\hline C & 1.5 & 0.0845 & 0.0016 & 0.1128 & 0.828 & 320 & 56.5 & 93.9 & 6.9 & 1.1 \\
\hline $\mathrm{D}$ & 2.0 & -0.2722 & 0.0016 & -1.1822 & 0.023 & 320 & -22.6 & 96.2 & 10 & 39 \\
\hline $\mathrm{E}$ & 3.0 & 0.0526 & 0.0016 & -0.1354 & 0.040 & 320 & 189.3 & 100.0 & 13 & 23 \\
\hline \multicolumn{3}{|c|}{ Integrated age $\pm 1 \sigma$} & $n=5$ & & \multirow{2}{*}{\multicolumn{2}{|c|}{$\begin{array}{l}1.047 \\
0.892\end{array}$}} & \multicolumn{2}{|c|}{$\mathrm{K} 2 \mathrm{O}=6.57 \%$} & 20.0 & 2.0 \\
\hline \multicolumn{2}{|c|}{ Plateau $\pm 1 \sigma$} & steps C-E & $n=3$ & $M S W D=1.76$ & & & & 85.2 & 6.9 & 1.1 \\
\hline \multicolumn{11}{|c|}{ Unit BA, Sanidine, $5.38 \mathrm{mg}, 4$} \\
\hline$x \mathrm{~A}$ & 0.5 & 8.127 & 0.0016 & 4.196 & 0.220 & 320 & 84.9 & 3.3 & 1089.4 & 7.0 \\
\hline$x B$ & 0.8 & 3.132 & 0.0016 & 1.917 & 0.281 & 320 & 82.0 & 7.5 & 405.1 & 4.8 \\
\hline$\times \mathrm{C}$ & 1.0 & 0.8164 & 0.0016 & 1.388 & 0.358 & 320 & 49.3 & 12.8 & 63.1 & 3.6 \\
\hline$x D$ & 1.2 & 0.4916 & 0.0016 & 1.091 & 0.378 & 320 & 33.4 & 18.5 & 25.5 & 3.3 \\
\hline$X \mathrm{E}$ & 1.5 & 0.5589 & 0.0016 & 1.272 & 0.612 & 320 & 31.9 & 27.6 & 27.8 & 2.2 \\
\hline$X \mathrm{~F}$ & 1.6 & 0.5625 & 0.0016 & 1.097 & 0.502 & 320 & 41.6 & 35.1 & 36.5 & 2.6 \\
\hline$x G$ & 1.8 & 0.3402 & 0.0016 & 0.5685 & 0.508 & 320 & 49.5 & 42.6 & 26.1 & 2.4 \\
\hline$X \mathrm{H}$ & 1.9 & 0.2262 & 0.0016 & 0.3867 & 0.540 & 320 & 47.7 & 50.7 & 16.5 & 2.2 \\
\hline 1 & 2.1 & 0.1669 & 0.0016 & 0.3575 & 0.686 & 320 & 33.6 & 60.9 & 8.5 & 1.8 \\
\hline $\mathrm{J}$ & 2.5 & 0.1362 & 0.0016 & 0.2625 & 0.640 & 320 & 39.6 & 70.4 & 8.1 & 1.9 \\
\hline $\mathrm{K}$ & 3.0 & 0.1754 & 0.0016 & 0.4222 & 1.98 & 320 & 25.7 & 100.0 & 6.81 & 0.75 \\
\hline \multicolumn{3}{|c|}{ Integrated age $\pm 1 \sigma$} & $n=11$ & & 6.71 & & $\mathrm{~K} 2 \mathrm{O}=$ & $=5.54 \%$ & 35.20 & 0.70 \\
\hline & $a u \pm 1 \sigma$ & steps I-K & $n=3$ & $M S W D=0.48$ & 3.310 & & & 49.3 & 7.16 & 0.65 \\
\hline
\end{tabular}




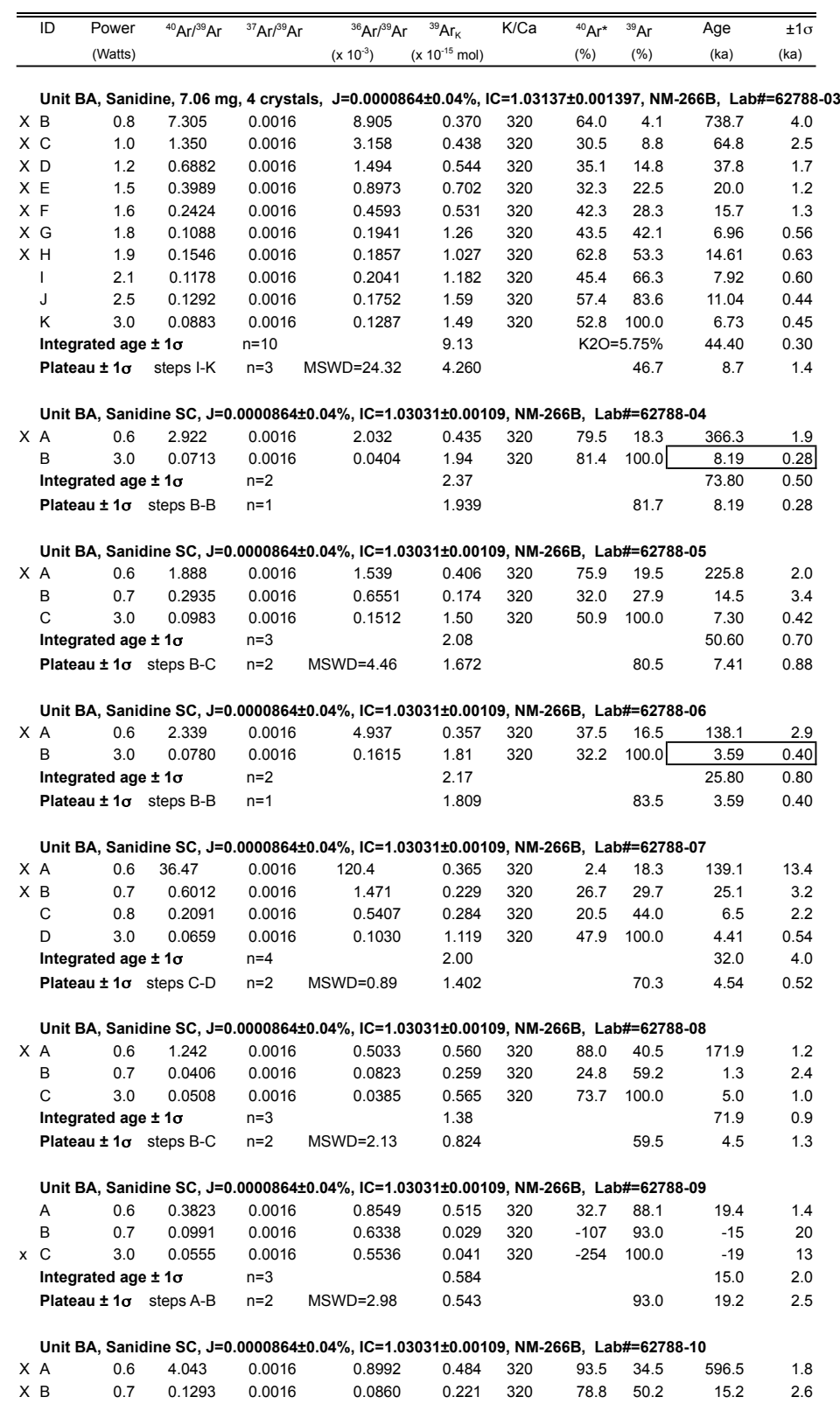




\begin{tabular}{|c|c|c|c|c|c|c|c|c|c|c|c|}
\hline & ID & $\begin{array}{l}\text { Power } \\
\text { (Watts) }\end{array}$ & ${ }^{40} \mathrm{Ar} /{ }^{39} \mathrm{Ar}$ & ${ }^{37} \mathrm{Ar} /{ }^{39} \mathrm{Ar}$ & $\begin{array}{l}\quad{ }^{36} \mathrm{Ar}{ }^{39} \mathrm{Ar} \\
\left(\times 10^{-3}\right)\end{array}$ & $\begin{array}{l}{ }^{2}{ }^{39} \mathrm{Ar}_{\mathrm{K}} \\
\left(\times 10^{-15} \mathrm{~mol}\right)\end{array}$ & $\mathrm{K} / \mathrm{Ca}$ & $\begin{array}{l}{ }^{40} \mathrm{Ar}^{*} \\
(\%)\end{array}$ & $\begin{array}{l}{ }^{39} \mathrm{Ar} \\
(\%)\end{array}$ & $\begin{array}{l}\text { Age } \\
\text { (ka) }\end{array}$ & $\begin{array}{l} \pm 1 \sigma \\
(\mathrm{ka})\end{array}$ \\
\hline & C & 3.0 & 0.1159 & 0.0016 & 0.2730 & 0.699 & 320 & 25.4 & 100.0 & 4.34 & 0.93 \\
\hline \multicolumn{4}{|c|}{ Integrated age $\pm 1 \sigma$} & $n=3$ & & 1.40 & & & & 210.2 & 1.0 \\
\hline \multicolumn{3}{|c|}{ Plateau $\pm 1 \sigma$} & steps C-C & $n=1$ & & 0.699 & & & 49.8 & 4.34 & 0.93 \\
\hline \multicolumn{12}{|c|}{ Unit BA, Sanidine SC, J=0.0000864 $\pm 0.04 \%, I C=1.03031 \pm 0.00109, N M-266 B$, Lab\#=62788-11 } \\
\hline \multirow{5}{*}{\multicolumn{2}{|c|}{$\begin{array}{r}X \\
\text { A } \\
B \\
C \\
\text { In } \\
P\end{array}$}} & 0.6 & 3.566 & 0.0016 & 7.645 & 0.206 & 320 & 36.6 & 5.7 & 205.9 & 4.5 \\
\hline & & 0.7 & 1.627 & 0.0016 & 5.111 & 0.138 & 320 & 6.7 & 9.5 & 17.3 & 6.1 \\
\hline & & 3.0 & 0.6758 & 0.0016 & 1.978 & 3.29 & 320 & 12.5 & 100.0 & 13.25 & 0.54 \\
\hline & & rated ag & $\pm 1 \sigma$ & $n=3$ & & 3.63 & & & & 24.30 & 0.70 \\
\hline & Plat & $a u \pm 1 \sigma$ & steps B-C & $n=2$ & $M S W D=0.43$ & 3.429 & & & 94.3 & 13.28 & 0.54 \\
\hline \multicolumn{12}{|c|}{ Unit BA, Sanidine SC, J=0.0000864 $\pm 0.04 \%, I C=1.03031 \pm 0.00109, N M-266 B$, Lab\#=62788-12 } \\
\hline \multirow{2}{*}{\multicolumn{2}{|c|}{$\begin{array}{r}X A \\
B\end{array}$}} & 0.6 & 0.7616 & 0.0016 & 0.9021 & 0.485 & 320 & 64.7 & 65.3 & 77.2 & 1.4 \\
\hline & B & 0.7 & & 0.0016 & -0.0209 & 0.133 & 320 & 117.8 & 83.2[ & 6.2 & 4.3 \\
\hline$x$ & & 3.0 & 0.2740 & 0.0016 & 0.5603 & 0.124 & 320 & 37.6 & 100.0 & 15.9 & 4.4 \\
\hline \multirow{2}{*}{\multicolumn{2}{|c|}{ In }} & rated ag & $\pm 1 \sigma$ & $n=3$ & & 0.742 & & & & 54.2 & 1.7 \\
\hline & & $\mathrm{au} \pm 1 \sigma$ & steps B-B & $n=1$ & & 0.133 & & & 17.9 & 6.2 & 4.3 \\
\hline
\end{tabular}

TC-24-13, Sanidine SC, $0.78 \mathrm{mg}, \mathrm{J}=0.0000645 \pm 0.05 \%, \mathrm{IC}=1.02237 \pm 0.00126, \mathrm{NM}-274 \mathrm{~B}$, Lab\#=63440-01

\begin{tabular}{|c|c|c|c|c|c|c|c|c|c|c|}
\hline A & 0.5 & 2.420 & 0.0280 & 8.039 & 0.342 & 18.2 & 1.6 & 39.5 & 4.6 & 3.3 \\
\hline B & 1.0 & 0.1010 & 0.0265 & 0.2021 & 0.332 & 19.2 & 38.1 & 77.8 & 4.2 & 1.5 \\
\hline & 3.0 & 0.0877 & 0.0324 & 0.5083 & 0.192 & 15.8 & -84.4 & 100.0 & -8.0 & 2.6 \\
\hline \multicolumn{3}{|c|}{ Integrated age $\pm 1 \sigma$} & $n=3$ & & 0.867 & 18.0 & $\mathrm{~K} 2 \mathrm{O}=$ & $=6.62 \%$ & 1.6 & 1.5 \\
\hline$P$ & $+1 \sigma$ & teps A-B & $n=2$ & $\mathrm{VD}=0.01$ & 0.675 & & & 77.8 & 4.3 & 1.4 \\
\hline
\end{tabular}

TC-24-13, Sanidine 9 crystals, $4.05 \mathrm{mg}, \mathrm{J}=\mathbf{0} .0000645 \pm 0.05 \%, \mathrm{IC}=1.02237 \pm 0.00126, \mathrm{NM}-274 \mathrm{~B}$, Lab\#=63440-02

$\begin{array}{rrrrrrrrrrr}\times \mathrm{A} & 0.4 & 7.014 & 0.0691 & 16.55 & 0.161 & 7.4 & 30.3 & 3.7 & 250.7 & 8.6 \\ \times & 0.6 & 1.716 & 0.0537 & 4.851 & 0.336 & 9.5 & 16.3 & 11.3 & 32.9 & 3.7\end{array}$

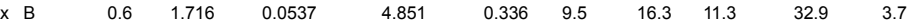

$\begin{array}{lllllllllll}\text { C } & 0.8 & 0.9578 & 0.0562 & 2.909 & 0.642 & 9.1 & 10.0 & 26.0 & 11.2 & 2.0\end{array}$

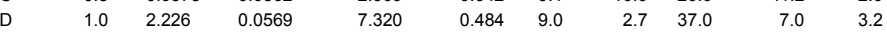

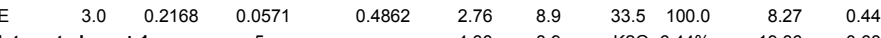

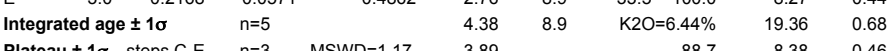

$\begin{array}{llll}\text { Plateau } \pm 1 \sigma & \text { steps } C-E \quad n=3 \quad M S W D=1.17 & 3.89 & 88.7\end{array}$

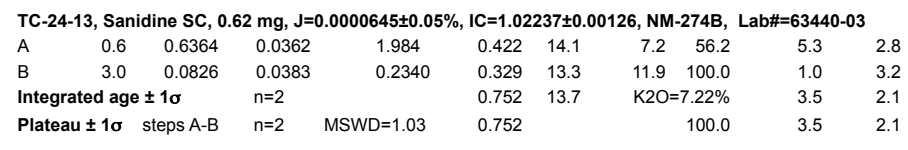

\begin{tabular}{|c|c|c|c|c|c|c|c|c|c|c|}
\hline A & 0.6 & 1.109 & 0.0438 & 3.206 & 0.267 & 11.7 & 14.3 & 42.4 & 18.6 & 4.2 \\
\hline B & 3.0 & 0.1033 & 0.0403 & 0.1524 & 0.362 & 12.7 & 56.2 & 100.0[ & 6.3 & 2.8 \\
\hline \multicolumn{3}{|c|}{ Integrated age $\pm 1 \sigma$} & $\mathrm{n}=2$ & & 0.630 & 12.2 & $\mathrm{~K} 2 \mathrm{O}=$ & $5.77 \%$ & 11.5 & 2.4 \\
\hline \multicolumn{2}{|c|}{ Step $B \pm 1 \sigma$} & step B & $n=1$ & NA & 0.362 & & & 57.6 & 6.3 & 2.8 \\
\hline
\end{tabular}

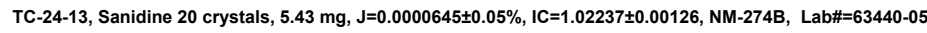

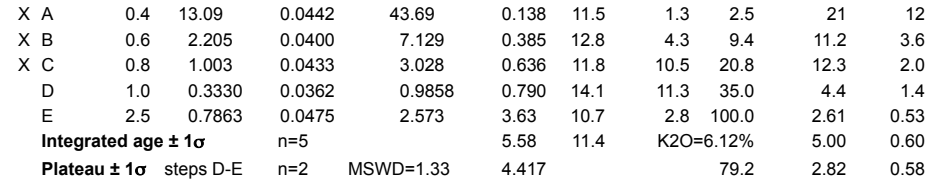

TC-24-13, Sanidine 20 crystals, $3.63 \mathrm{mg}, \mathrm{J}=0.0000645 \pm 0.05 \%, \mathrm{IC}=1.02237 \pm 0.00126$, NM-274B, Lab\#=63440-06

$\begin{array}{llllllrrrrr}\text { A } & 0.4 & 4.122 & 0.0517 & 13.87 & 0.221 & 9.9 & 0.4 & 5.3 & 2.1 & 6.1 \\ \text { B } & 0.6 & 1.483 & 0.0497 & 4.898 & 0.692 & 10.3 & 2.2 & 22.0 & 3.8 & 2.1\end{array}$ 


\begin{tabular}{|c|c|c|c|c|c|c|c|c|c|c|}
\hline ID & $\begin{array}{l}\text { Power } \\
\text { (Watts) }\end{array}$ & ${ }^{40} \mathrm{Ar} /{ }^{39} \mathrm{Ar}$ & ${ }^{37} \mathrm{Ar} /{ }^{39} \mathrm{Ar}$ & $\begin{array}{l}{ }^{36} \mathrm{Ar} r^{39} \mathrm{Ar} \\
\left(\times 10^{-3}\right)\end{array}$ & $\begin{array}{c}{ }^{39} \mathrm{Ar}_{\mathrm{K}} \\
\left(\times 10^{-15} \mathrm{~mol}\right)\end{array}$ & $\mathrm{K} / \mathrm{Ca}$ & $\begin{array}{l}{ }^{40} \mathrm{Ar}^{*} \\
(\%)\end{array}$ & $\begin{array}{l}{ }^{39} \mathrm{Ar} \\
(\%) \\
\end{array}$ & $\begin{array}{l}\text { Age } \\
\text { (ka) }\end{array}$ & $\begin{array}{c} \pm 1 \sigma \\
(\mathrm{ka})\end{array}$ \\
\hline C & 0.8 & 0.4053 & 0.0420 & 1.320 & 0.694 & 12.1 & 2.7 & 38.7 & 1.3 & 1.7 \\
\hline D & 1.0 & 0.4924 & 0.0500 & 1.680 & 0.330 & 10.2 & -1.6 & 46.7 & -0.9 & 3.3 \\
\hline E & 1.5 & 0.2656 & 0.0477 & 0.7729 & 1.58 & 10.7 & 12.9 & 84.9 & 3.9 & 0.8 \\
\hline $\mathrm{F}$ & 3.0 & 0.7305 & 0.0485 & 2.487 & 0.629 & 10.5 & -1.1 & 100.0 & -1.0 & 2.1 \\
\hline \multicolumn{3}{|c|}{ Integrated age $\pm 1 \sigma$} & $n=6$ & & 4.15 & 10.7 & $\mathrm{~K} 2 \mathrm{O}=$ & $6.81 \%$ & 2.24 & 0.76 \\
\hline \multicolumn{2}{|c|}{ Plateau $\pm 1 \sigma$} & teps A-F & $n=6$ & $M S W D=1.52$ & 4.15 & & & 100.0 & 2.95 & 0.77 \\
\hline
\end{tabular}

TC-27-13, Sanidine SC, $2.89 \mathrm{mg}, \mathrm{J}=\mathbf{0} .0000647 \pm 0.04 \%, \mathrm{IC}=1.02237 \pm 0.00126$, NM-274B, Lab\#=63441-01

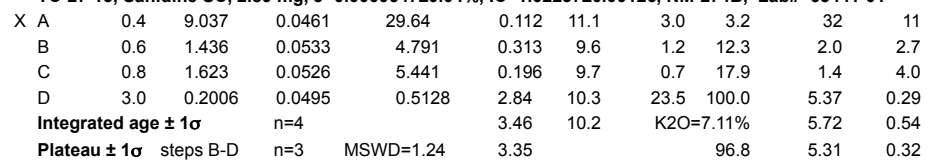

TC-27-13, Sanidine 20 crystals, $4.28 \mathrm{mg}, \mathrm{J}=0.0000647 \pm 0.04 \%, \mathrm{IC}=1.02237 \pm 0.00126, \mathrm{NM}-274 \mathrm{~B}$, Lab\#=63441-02

\begin{tabular}{|c|c|c|c|c|c|c|c|c|c|c|}
\hline$x A$ & 0.4 & 9.172 & 0.0498 & 28.93 & 0.159 & 10.2 & 6.8 & 3.1 & 73.6 & 9.5 \\
\hline$x B$ & 0.6 & 2.313 & 0.0535 & 7.285 & 0.342 & 9.5 & 6.8 & 9.9 & 18.6 & 4.0 \\
\hline C & 0.6 & 1.199 & 0.0572 & 3.786 & 0.188 & 8.9 & 6.5 & 13.6 & 9.1 & 6.3 \\
\hline D & 1.0 & 1.395 & 0.0521 & 4.442 & 0.644 & 9.8 & 5.7 & 26.2 & 9.4 & 2.1 \\
\hline $\mathrm{E}$ & 1.5 & 0.6130 & 0.0513 & 1.783 & 1.164 & 9.9 & 13.6 & 49.2 & 9.8 & 1.1 \\
\hline $\mathrm{F}$ & 3.0 & 0.3589 & 0.0499 & 0.9972 & 2.58 & 10.2 & 17.2 & 100.0 & 7.17 & 0.52 \\
\hline \multicolumn{3}{|c|}{ d age $\pm 1 \sigma$} & $n=6$ & & 5.08 & 10.0 & $\mathrm{~K} 2 \mathrm{O}=$ & $7.04 \%$ & 10.97 & 0.65 \\
\hline & $1 \sigma$ & eps C-F & $n=4$ & $N D=1.70$ & 4.58 & & & 90.1 & 7.72 & 0.60 \\
\hline
\end{tabular}

128

129

\begin{tabular}{|c|c|c|c|c|c|c|c|c|c|c|}
\hline \multirow[t]{2}{*}{ ID } & Power & ${ }^{40} \mathrm{Ar} /{ }^{39} \mathrm{Ar}$ & ${ }^{37} \mathrm{Ar} /{ }^{39} \mathrm{Ar}$ & ${ }^{36} \mathrm{Ar} /{ }^{39} \mathrm{Ar}$ & ${ }^{39} \mathrm{Ar}_{\mathrm{K}}$ & $\mathrm{K} / \mathrm{Ca}$ & ${ }^{40} \mathrm{Ar}^{*}$ & ${ }^{39} \mathrm{Ar}$ & Age & \pm 10 \\
\hline & (Watts) & & & $\left(\times 10^{-3}\right)$ & $\left(\times 10^{-15} \mathrm{~mol}\right)$ & & $(\%)$ & (\%) & (ka) & (ka) \\
\hline \multicolumn{11}{|c|}{ TC-27-13, Sanidine SC, $2.25 \mathrm{mg}, \mathrm{J}=0.0000647 \pm 0.04 \%, \mathrm{IC}=1.02237 \pm 0.00126, \mathrm{NM}-274 \mathrm{~B}$, Lab\#=63441-03 } \\
\hline$x \mathrm{~A}$ & 0.5 & 11.98 & 0.0343 & 39.11 & 0.157 & 14.9 & 3.5 & 5.3 & 49 & 11 \\
\hline B & 1.0 & 0.5498 & 0.0317 & 1.605 & 1.66 & 16.1 & 13.0 & 61.1 & 8.34 & 0.78 \\
\hline C & 3.0 & 0.1154 & 0.0335 & 0.2181 & 1.158 & 15.2 & 42.6 & 100.0 & 5.43 & 0.92 \\
\hline \multicolumn{3}{|c|}{ Integrated age $\pm 1 \sigma$} & $n=3$ & & 2.98 & 15.7 & $\mathrm{~K} 2 \mathrm{O}=$ & $=7.86 \%$ & 9.38 & 0.80 \\
\hline $\mathrm{Pla}$ & $u \pm 1 \sigma$ & steps B-C & $n=2$ & $M S W D=5.84$ & 2.821 & & & 94.7 & 7.1 & 1.4 \\
\hline
\end{tabular}

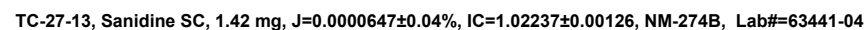

\begin{tabular}{|c|c|c|c|c|c|c|c|c|c|c|}
\hline$X \mathrm{~A}$ & 0.5 & 2.755 & 0.0196 & 7.971 & 0.252 & 26.0 & 14.3 & 14.8 & 46.7 & 4.9 \\
\hline B & 1.0 & 0.14 & 0.0214 & 0.3350 & 1.123 & 23.9 & 27.9 & 80.9 & 4.5 & 9 \\
\hline c & 3.0 & 0.0655 & 0.0240 & 0.1554 & 0.325 & 21.3 & 23.9 & 100.0 & 1.6 & 1 \\
\hline \multicolumn{3}{|c|}{ Integrated age $\pm 1 \sigma$} & $n=3$ & & 1.70 & 23.6 & $\mathrm{~K} 2 \mathrm{O}$ & $=7.11 \%$ & 10.2 & 1.3 \\
\hline $\mathrm{Pla}$ & $1 \sigma$ & eps B-C & $n=2$ & $\mathrm{D}=0.76$ & 1.448 & 23.275 & .828 & 85.2 & 4.2 & \\
\hline
\end{tabular}

130 


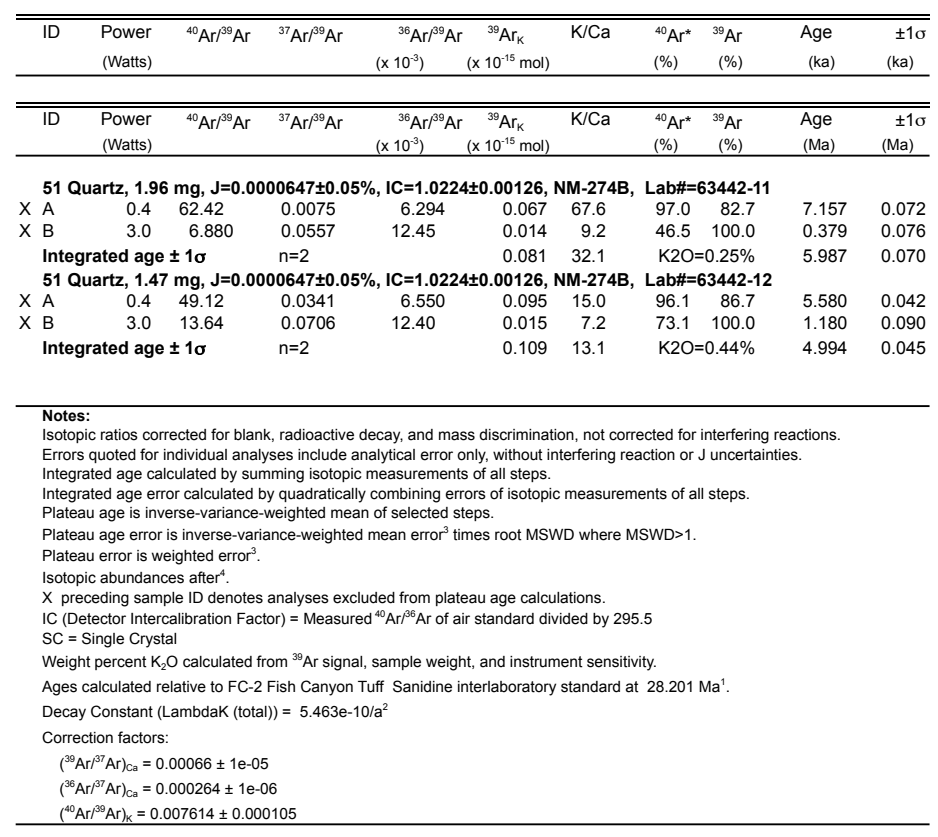

131

132

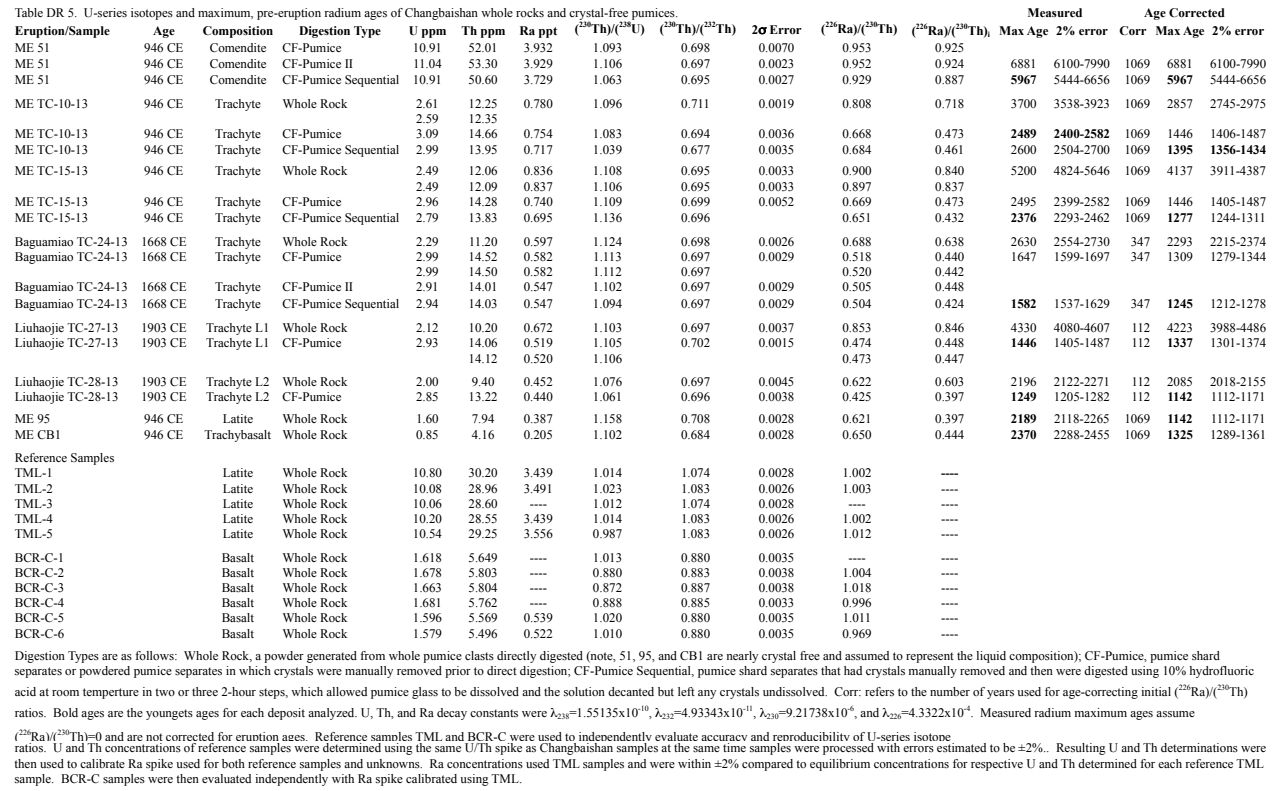




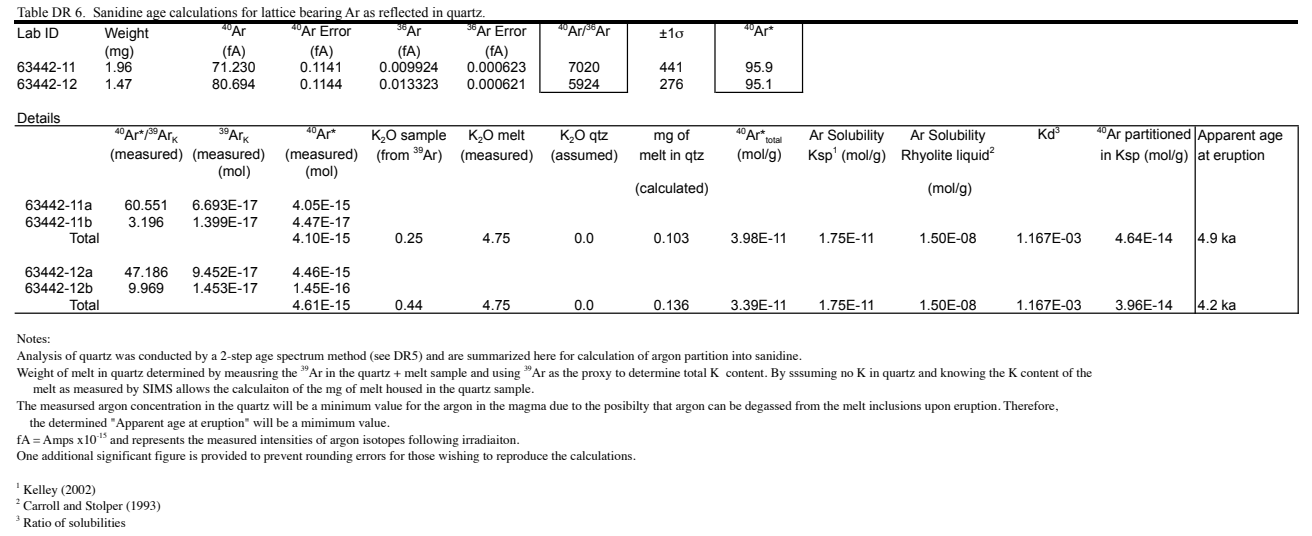

\section{Supplementary References}

137 1. Taylor, J.R., 1982, An Introduction to Error Analysis: The Study of Uncertainties 138 in Physical Measurements: Univ. Sci. Books, Mill Valley, Calif., 270 p.

139 2. Steiger, R.H. and Jäger, E., 1977, Subcommission on geochronology: Convention 140 on the use of decay constants in geo- and cosmochronology: EPSL, v. 36, p. 359$141 \quad 362$.

142 3. Kelley, S., 2002, Excess argon in K-Ar and Ar-Ar geochronology: Chemical 143 Geology, v. 188, p. 1-22.

144 4. Carroll, M.R. and Stolper, E.M., 1993, Noble gas solubilities in silicate melts and 145 glasses - new experimental results for argon and the relationship between 146 solubility and ionic porosity: Geochim. Cosmochim. Acta, v. 57, p. 5039-5051. 